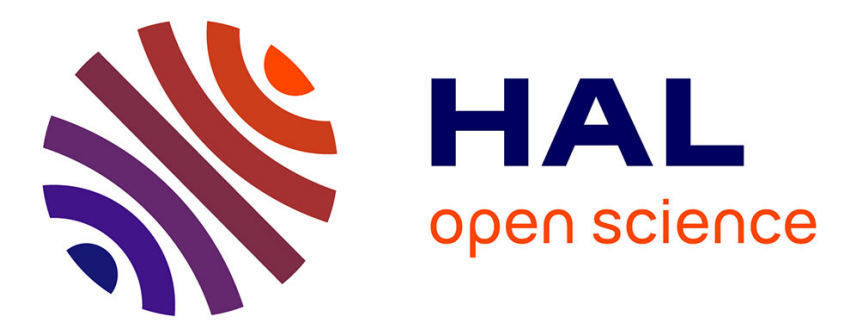

\title{
Dispersive characteristics of non-linear waves propagating and breaking over a mildly sloping laboratory beach
}

Kévin Martins, Philippe Bonneton, Hervé Michallet

\section{To cite this version:}

Kévin Martins, Philippe Bonneton, Hervé Michallet. Dispersive characteristics of non-linear waves propagating and breaking over a mildly sloping laboratory beach. Coastal Engineering, 2021, 167, pp.103917. 10.1016/j.coastaleng.2021.103917 . hal-03215337

\section{HAL Id: hal-03215337 \\ https://hal.science/hal-03215337}

Submitted on 3 May 2021

HAL is a multi-disciplinary open access archive for the deposit and dissemination of scientific research documents, whether they are published or not. The documents may come from teaching and research institutions in France or abroad, or from public or private research centers.
L'archive ouverte pluridisciplinaire HAL, est destinée au dépôt et à la diffusion de documents scientifiques de niveau recherche, publiés ou non, émanant des établissements d'enseignement et de recherche français ou étrangers, des laboratoires publics ou privés. 


\title{
Dispersive characteristics of non-linear waves propagating and breaking over a mildly sloping laboratory beach
}

\author{
Kévin Martins ${ }^{\mathrm{a}, *}$, Philippe Bonneton ${ }^{\mathrm{a}}$, Hervé Michallet ${ }^{\mathrm{b}}$ \\ ${ }^{a}$ UMR 5805 EPOC, CNRS - University of Bordeaux, Allée Geoffroy Saint-Hilaire, F-33615 Pessac, France \\ ${ }^{b}$ UMR 5519 LEGI, CNRS - University Grenoble Alpes, CS40700, 38058 Grenoble, France
}

\begin{abstract}
The dispersive characteristics of unidirectional irregular waves propagating and breaking over a mildly sloping beach are examined using a highly-resolved laboratory dataset. Cross-spectral analyses are used to determine the cross-shore evolution of (single-valued) dominant wavenumber $\kappa$ and phase velocity $c$ spectra, and lead to the identification of four different regimes of propagation: I - a linear regime where short waves mostly propagate as free components; II - a shoaling regime where non-linear effects at high harmonics are significant but primary components follow the linear wave dispersion relation; III - a shoaling regime near the mean breaking point location, where amplitude dispersion effects at primary components are important; IV - a surf zone regime, where all components propagate slightly faster than non-dispersive shallow water waves. Bispectral analyses performed onshore of the shoaling region show that the presence of forced energy at high harmonics, which originate from non-linear interactions between triads of frequencies, are responsible for the deviations of wavenumber and phase velocity spectral estimates from predictions by the linear dispersion relation, confirming the findings from previous field-based studies. A Boussinesq approximation of the non-linear energy exchanges between triads is then used to quantify the relative amount of forced energy at high harmonics and explain the differences in dispersion properties observed in the shoaling region between broad and narrow-band spectra. Larger relative amounts of forced energy at high frequencies, which suggest more efficient non-linear energy transfers, are found to be associated with larger deviations of dominant $\kappa$ and $c$ from predictions by the linear dispersion relation.
\end{abstract}

\section{Introduction}

Wind-generated surface gravity waves (hereafter short waves) are the principal driver of nearshore dynamics. Close to shore, short waves eventually break and through this process, they enhance the vertical 4 and horizontal mixing of the water column (e.g., Ting and Kirby, 1996; Drazen and Melville, 2009; Clark 5 et al., 2012), drive a setup near the shoreline (e.g., Longuet-Higgins and Stewart, 1964; Stive and Wind, 6 1982) and control the nearshore circulation at various temporal scales (e.g., Svendsen, 1984; Peregrine and 7 Bokhove, 1998; Bühler and Jacobson, 2001; Bonneton et al., 2010; Castelle et al., 2016). At first order, 8 linear wave theory correctly predicts a number of physical processes associated with the propagation of 9 short waves, such as the refraction or shoaling of directionally spread seas (e.g. Longuet-Higgins, 1956; 10 Guza and Thornton, 1980; Elgar et al., 1990, and many others). However, as waves shoal and interact

\footnotetext{
* Corresponding author

Email address: kevin.martins@u-bordeaux.fr (Kévin Martins)
} 
with a sloping, mobile bed, non-linear processes become dominant. These are responsible for the changes observed in wave shape, from nearly symmetric to more (horizontally) skewed in the shoaling region and more (vertically) asymmetric prior to breaking and in the surf zone (e.g. Elgar and Guza, 1985a,b; Doering and Bowen, 1995; Michallet et al., 2011; Rocha et al., 2017). Non-linear effects not only affect the sea surface elevation but also the near-bottom wave orbital velocities and thus play a crucial role in short- and long-term beach morphodynamics (Doering and Bowen, 1986, 1995; Hoefel and Elgar, 2003; Berni et al., 2013; van der Zanden et al., 2017).

Weakly non-linear triad interactions (e.g. Phillips, 1960; Freilich et al., 1984; Elgar and Guza, 1985a) occurring as short waves propagate landward over a sloping bottom are responsible for these changes in the wave field. The interaction of two primary components of frequencies $f_{1}$ and $f_{2}$ excite a secondary component $f^{\prime}$ (either sum $f_{1}+f_{2}$ or difference $f_{1}-f_{2}$ ), which is bound to the statistically independent primary components. As such, the bound wave component $f^{\prime}$ does not follow the linear wave dispersion relation (Phillips, 1960; Longuet-Higgins and Stewart, 1962; Freilich et al., 1984). As opposed to non-linear resonant interactions between quadruplets in deep water (Hasselmann, 1962), which require very large distances to be effective, non-linear coupling between triads in nearshore areas are non- or near-resonant and can be very efficient in transforming incident wave spectra over just few typical wavelengths (e.g., see Freilich et al., 1984, and the references therein). As both forced (or 'bound') and free components of directionally spread seas can co-exist in a wave field, there is no longer a unique relation between a frequency and wavenumber (e.g. Herbers and Guza, 1994). When forced components dominate over a region of the spectrum, large deviations from predictions by the linear wave dispersion relation can be observed in (single-valued) dominant wavenumber and phase velocity spectra (Thornton and Guza, 1982; Freilich et al., 1984; Elgar and Guza, 1985b). In particular near the breaking point or in the surf zone, most wave components of a typical sea-surface spectrum travel at the speed of non-dispersive shallow-water waves (e.g., see Thornton and Guza, 1982; Elgar and Guza, 1985b; Catalán and Haller, 2008; Tissier et al., 2011), which is due to the dominance of amplitude dispersion effects over frequency ones (Herbers et al., 2002). As noted by Laing (1986), the deviations of measured wave phase speed from predictions by the linear dispersion relation, discussed here for nearshore waves, are quite analogous to those observed in growing seas (e.g., see Ramamonjiarisoa and Coantic, 1976; Mitsuyasu et al., 1979; Crawford et al., 1981; Donelan et al., 1985). In such conditions, growing short-wave fields are dominated by modulated trains of finite amplitude waves to which high-frequency components are bound (Lake and Yuen, 1978; Coantic et al., 1981).

In practice, knowledge on the spatial structure of the wave field is generally lacking and the presence of forced energy is therefore difficult to quantify. As forced components at high harmonics are characterised by lower wavenumbers than free components of the same frequency, large errors from depth-inversion algorithms based on the linear wave dispersion relation can be expected in regions where non-linear effects are important (e.g., see Holland, 2001; Brodie et al., 2018). The over-predictions of the dominant wavenumbers at high frequencies also explain the commonly reported 'blow-up' when reconstructing the free surface elevation from sub-surface pressure measurements with the linear dispersion relation (Bonneton and Lannes, 2017; Bonneton et al., 2018; Mouragues et al., 2019; Martins et al., 2020b). This is related to the fact 
that forced high harmonics are much less attenuated across the vertical than free components of the same frequency (e.g. Herbers and Guza, 1991; Herbers et al., 1992). Nonetheless, most field-based studies on non-linear wave transformation in the shoaling region employed sub-surface hydrodynamic data (whether pressure or orbital wave velocity), not corrected or corrected for depth-attenuation using the linear wave dispersion relation. Field-based studies on wave non-linearity also suffer from other limitations such as a poor spatial resolution and the distance over which waves can be studied. In particular, the cross-shore location where non-linear effects at high harmonics become predominant remains largely unknown.

The present paper uses a high-resolution laboratory dataset (GLOBEX, see Ruessink et al., 2013) to study the dispersive properties of irregular waves propagating and breaking over a mildly sloping beach. Besides confirming past findings, the GLOBEX dataset stands out from previously-published field observations for several reasons. The free surface is directly measured with wave gauges and it is highly-resolved in space (several points per wavelength at any stage of propagation). The former aspect removes uncertainties currently existing on energy levels at high harmonics as measured in the field by sub-surface pressure sensors, and where the choice of the surface elevation reconstruction method has a strong influence (Bonneton et al., 2018; Mouragues et al., 2019; Martins et al., 2020b). Furthermore, the experiments considered unidirectional irregular waves, which removes uncertainty about directional effects. In section 2 , the high spatial and temporal resolution experimental dataset collected during GLOBEX is briefly presented. Section 3 introduces the cross-spectral and bispectral analysis techniques and describes the weakly non-linear numerical approach employed here for predicting the cross-shore evolution of energy spectra. In Section 4 , the cross-spectral analysis is performed on the surface elevation data from adjacent wave gauges to extract dominant wavenumber spectra $\kappa(f)$, phase velocity spectra $c(f)$ and their evolution across the entire wave flume. From this analysis, we identify four regimes of propagation ranging from a linear up to a surf zone situation, where wavenumber and phase velocity spectra display specific characteristics. In section 5 , the bispectral analysis is used to quantify non-linear energy transfers towards harmonics, which play a fundamental role in the patterns observed at high frequencies in wavenumber and phase velocity spectra. The dominant wavenumber is shown to vary with the amount of forced energy at a particular frequency, with larger deviations from the linear wave dispersion expected for higher forced-to-free energy ratios. Finally, section 6 briefly discusses the results and provides the concluding remarks of this study.

\section{Experimental dataset}

The Gently sLOping Beach Experiment (GLOBEX) project was performed in a 110-m-long, 1-m-wide, and 1.2-m-high wave flume, located in the Scheldegoot in Delft, the Netherlands (Ruessink et al., 2013). The experiments aimed at collecting high-resolution data of free surface elevation and current velocities in order to study infragravity wave dynamics and short-wave propagation and non-linearities (e.g., see de Bakker et al., 2015; Tissier et al., 2015; Rocha et al., 2017). A combination of 21 capacitance and resistance-type of wave gauges sampling at $128 \mathrm{~Hz}$ were deployed along the low-sloping 1:80 concrete beach to measure the free surface elevation (see Fig. 1). 18 of these wave gauges were mounted on movable trolleys, which were repositioned after the repetition of each wave test in order to reach the desired spatial resolution (10 repetitions per test). Similarly, five electromagnetic current meters were fixed to the trolleys 
and allowed the collection of current velocities at numerous locations across the wave flume (the data from these current meters is not used here). This spatial resolution makes the GLOBEX dataset unique as it allows to characterize and quantify non-linearities at various stages of the waves propagation (from a linear situation up to the surf zone). The wave paddle steering signals included second-order wave generation and the paddle was equipped with an Active Reflection Compensation system to absorb long waves radiated from the beach. The water depth at the wave paddle was $0.85 \mathrm{~m}$ for all tests.

The present study uses free surface elevation measurements from the 70-min-long irregular wave tests of the A series (A1, A2, A3, see Ruessink et al., 2013). During this series of tests, JONSWAP spectra were imposed, covering moderate to energetic and broad to narrow-banded sea wave conditions, see table 1. The wave conditions along the flume for these runs are displayed in Fig. 2, through a range of second and third-order wave parameters. Fig. 2a first shows the root-mean square wave height $H_{r m s}$ computed as $\left(8 \overline{\zeta^{2}}\right)^{1 / 2}$ where $\zeta$ is the free surface elevation and the overbar denotes the time-averaged operator. Note that $\zeta$ was high-pass filtered using a cutoff frequency at $0.6 f_{p}$, with $f_{p}$ the peak wave frequency, so that the bulk parameters shown in Fig. 2 are computed on the short-wave frequency band only. As conditions were more energetic during A2, the mean breaking point was located farther seaward than during A1 and A3. In the inner surf zone, waves were found to be depth-limited during all runs, which is also evidenced by the near-constant values reached by the non-linearity parameter $\epsilon=H_{r m s} / \sqrt{2} h$ in this region of the wave flume $(\epsilon \sim 0.3$, see Fig. $2 \mathrm{~b})$, where $h$ is the mean water depth. Fig. 2c-d show the short-wave skewness $S_{k}$ and asymmetry $A_{s}$ computed respectively as

$$
\begin{gathered}
S_{k}=\frac{\overline{(\zeta-\bar{\zeta})^{3}}}{{\overline{(\zeta-\bar{\zeta})^{2}}}^{3 / 2}} . \\
A_{s}=-\frac{\overline{(\mathcal{H}(\zeta-\bar{\zeta}))^{3}}}{{\overline{(\zeta-\bar{\zeta})^{2}}}^{3 / 2}},
\end{gathered}
$$

where $\mathcal{H}\{\cdot\}$ is the Hilbert transform. $S_{k}$ and $A_{s}$ are measures of wave asymmetry along the horizontal and vertical axes respectively and also inform on the energy content at high harmonics (Elgar, 1987; Martins et al., 2020b; de Wit et al., 2020). For the same imposed wave height, A1 and A3 differed in the shoaling region, with a more intense shoaling process noted during A3 (Fig. 2a and 2c), as expected for longer waves. This is also explained by the narrower spectrum imposed during A3, which favoured non-linear energy transfers to higher harmonics as compared to A1 (de Bakker et al., 2015). Short waves remain (vertically) symmetric $\left(A_{s} \sim 0\right)$ until the location where the largest waves start breaking. Short-wave asymmetry is maximal in the inner surf zone, where short waves pitch forward and display the commonly observed sawtooth-like shape. Finally, the Ursell number $U_{r}$ is shown in Fig. 2e. Here, $U_{r}$ is defined as the ratio between the non-linearity $\epsilon$ and dispersion $\mu=\left(\kappa_{p} h\right)^{2}$ parameters, where $\kappa_{p}$ is the peak wavenumber given by the linear wave dispersion relation.

\section{Methods}

\subsection{Computation of wavenumber and phase velocity spectra}

Cross-spectral analysis between adjacent wave gauges is used to compute the dominant wavenumber and phase velocity spectra across the wave flume. As this approach provides phase differences (or delay) 
between two signals in the frequency domain (e.g., see Ochi, 1998), it has been successfully used in the past to study, in both the laboratory and field, the dispersive properties of ocean waves propagating in deep (e.g., see Ramamonjiarisoa and Coantic, 1976; Mitsuyasu et al., 1979) and intermediate to shallow waters (Thornton and Guza, 1982; Freilich et al., 1984; Elgar and Guza, 1985b).

Let $C_{x_{1}, x_{2}}$ denote the cross-spectrum computed with surface elevation timeseries from two gauges located at positions $x_{1}$ and $x_{2}$. The coherence $\operatorname{coh}(\mathrm{f})$ and phase $\phi(f)$ spectra computed between $x_{1}$ and $x_{2}$ are then respectively given by

$$
\begin{aligned}
\operatorname{coh}_{\mathrm{x}_{1}, \mathrm{x}_{2}}(\mathrm{f}) & =\left[\frac{C_{x_{1}, x_{2}}(f) C_{x_{1}, x_{2}}^{*}(f)}{C_{x_{1}, x_{1}}(f) C_{x_{2}, x_{2}}(f)}\right]^{1 / 2} \\
\phi_{x_{1}, x_{2}}(f) & =\arctan \left[\frac{\operatorname{Im}\left\{C_{x_{1}, x_{2}}(f)\right\}}{\operatorname{Re}\left\{C_{x_{1}, x_{2}}(f)\right\}}\right],
\end{aligned}
$$

where $\operatorname{Re}\{\cdot\}$ and $\operatorname{Im}\{\cdot\}$ are the real and imaginary parts of the cross-spectra respectively and * denotes the complex conjugate. As shown in Fig. 3a with an example of cross-spectral analysis performed during A2 in the deepest region of the flume $(x \sim 10 \mathrm{~m})$, the phase spectra $\phi(f)$ provides a phase lag per frequency that is bounded between $-\pi / 2$ and $\pi / 2$. The time delay (in sec) per frequency is obtained from the unwrapped phase $\phi^{u n w}$ which, in the case of progressive waves propagating in one dimension, is easily retrieved from the phase jumps (e.g., see Fig. 3a). The wavenumber $\kappa(f)$ and (cross-shore) phase velocity $c(f)$ spectra are then readily computed as

$$
\begin{aligned}
\kappa(f) & =\phi_{x_{1}, x_{2}}^{u n w}(f) / \Delta x \\
c(f) & =2 \pi f \Delta x / \phi_{x_{1}, x_{2}}^{u n w}(f),
\end{aligned}
$$

where $\Delta x$ is the spacing between the two wave gauges. As in Herbers et al. (2002), $\kappa$ refers to the single-valued wavenumber modulus, representative of a mixed sea-state composed of both free and forced components $(i . e .$, for a given angular frequency $\omega=2 \pi f$, energy in the $(\omega, k)$ space is spread across several wavenumbers $k$ ). In practice, $\kappa$ and $c$ provide estimates at $x=\left(x_{1}+x_{2}\right) / 2$ of the dominant wavenumber (in an energy-averaged sense) and the corresponding propagation speed respectively, as shown by the analysis on synthetic data in Appendix A. This aspect is also discussed in Section 5. Cross-spectra were computed using Welch's method and 63 Hann-windowed records of 128 seconds, which were overlapping by $50 \%$. This resulted in each spectral estimate having approximately 70 equivalent degrees of freedom and a spectral resolution of $0.008 \mathrm{~Hz}$.

As observed by many authors in the past, the coherence spectra at high harmonics were found to be quite sensitive to the spacing between the two adjacent wave gauges. This is especially true in the deepest parts of the wave flume, where energy levels at these frequencies are quite low and the spacing between the two gauges can represent several wavelengths. An example is provided in Fig. 3b, which shows the coherence spectra computed over the flat section of the flume during A2 with $\Delta x=0.93 L_{p}, L_{p}$ being the peak wavelength given by linear wave theory. The coherence remains very high (coh $>0.95)$ at frequencies between 0.6 and $1.5 f_{p}$, which explain over $86 \%$ of the short-wave variance at this location. However, valleys in the coherence can be observed around $2.5 f_{p}$ for this particular spacing configuration and the coherence weakens quickly after $2.8 f_{p}$ (Fig. 3b). Since using a different spacing slightly shifts the coherence 'valleys', several combinations of wave gauges and spacing were used to obtain spectral estimates at a single location 
with coherence higher than typically 0.5 at all frequencies (similar idea as that used by Herbers et al., 2002 , with their field observations). After the removal of data associated with a coherence inferior to 0.5 $\left(\operatorname{coh}^{2} \gtrsim 0.25\right.$ in Fig. 3b), spectral estimates were ensemble-averaged. When non-linearities are strong, such as near the breaking point or in the surf zone, the coherence remains high up to $4-5 f_{p}$, as long as $\Delta x$ is taken sufficiently small (typically $0.2-0.4 L_{p}$ in those regions). In such cases the ensemble-averaging procedure is not really necessary but it was used all along the flume for consistency in the analysis.

\subsection{Computation of bispectra}

The power bispectrum of discretely sampled data corresponds to a representation in the frequency domain of its third-order cumulant or moment. As it provides information on the strength of the phase coupling between triads of frequencies $f_{1}, f_{2}$ and $f_{1}+f_{2}$, the bispectrum is a useful and powerful tool for studying non-linear phenomena in ocean waves (Hasselmann et al., 1963; Elgar and Guza, 1985a). Here, we use the definition given by Kim and Powers (1979):

$$
B\left(f_{1}, f_{2}\right)=\mathcal{E}\left[A\left(f_{1}\right) A\left(f_{2}\right) A^{*}\left(f_{1}+f_{2}\right)\right],
$$

where $A(f)$ are the complex Fourier coefficients and $\mathcal{E}$ is an expected, or ensemble-average, value. Similar to the cross-spectrum phase, the biphase is obtained from the bispectrum as

$$
\beta\left(f_{1}, f_{2}\right)=\arctan \left[\frac{\operatorname{Im}\left\{B\left(f_{1}, f_{2}\right)\right\}}{\operatorname{Re}\left\{B\left(f_{1}, f_{2}\right)\right\}}\right] .
$$

It is particularly insightful to recast the biphase as a function of the Fourier coefficients phases $\theta(f)$ : $\beta\left(f_{1}, f_{2}\right)=\theta\left(f_{1}\right)+\theta\left(f_{2}\right)-\theta\left(f_{1}+f_{2}\right)$ (e.g., see Kim et al., 1980; Elgar and Guza, 1985a). The amount of energy transfer between near-resonant components depends on their relative phases, whose information is contained in the biphase (Hasselmann et al., 1963; Kim et al., 1980).

Bispectra were computed on the free surface elevation signals down-sampled to $16 \mathrm{~Hz}$ by averaging estimates from 126 Hann-windowed records of 128 seconds, which were overlapping by $75 \%$. Statistical stability was increased by merging estimates over three frequencies (e.g., see Elgar and Guza, 1985a) yielding approximately 205 equivalent degrees of freedom, and a spectral resolution of $0.023 \mathrm{~Hz}$. A validation of the bispectra computation is provided in Appendix B, which compares surface elevation third-order moments computed across the whole wave flume via bispectral (Elgar and Guza, 1985a; Elgar, 1987) and statistical definitions (Eq. 1 and 2).

\subsection{Non-linear energy transfers}

In the present study, we are principally interested in the growth of high harmonics due to non-linear energy exchanges between triads and the effect of these forced components on the dispersive properties of shoaling waves. To quantify the relative importance of forced energy at high harmonics, we represent these exchanges with a Boussinesq approximation in a spectral energy balance equation, following a similar approach as developed in Herbers et al. (2000). Such Boussinesq approximation has already demonstrated its potential to predict, in a very computationally efficient manner, the cross-shore evolution of spectral components in the nearshore (Herbers and Burton, 1997; Norheim et al., 1998; Herbers et al., 2000; de Bakker et al., 2015; Padilla and Alsina, 2017). Note that the Boussinesq approximation (detailed below) restricts the present modelling approach to A2 and A3 since conditions during A1 are too dispersive. 
For unidirectional waves propagating shoreward on an alongshore-uniform beach and assuming a weak reflection at the shoreline, a balance between the cross-shore gradient of the energy flux spectrum $F(f)$, a source term $S_{n l}(f)$ quantifying the non-linear energy exchanges between triads, and a dissipation term $S_{d i s}(f)$ reads (e.g., Eq. 1 of Herbers et al., 2000)

$$
\frac{\partial F(f)}{\partial x}=S_{n l}(f)+S_{d i s}(f) .
$$

Breaking processes are ignored so that the dissipation term $S_{d i s}$ reduces to the energy losses by bottom friction $S_{f r}$, here simply modelled after Thornton and Guza (1983):

$$
S_{f r}(f)=\rho c_{f} \frac{1}{6 \pi}\left(\frac{2 \pi f}{\sinh |k| h} H\right)^{3},
$$

where $h$ is the mean water depth and $|k|$ is the wavenumber modulus obtained from the linear wave dispersion relation (hereafter denoted $\kappa_{L}$, see Section 4). The friction coefficient $c_{f}$ was set to 0.0055 after calibration in the deepest section of the wave flume.

Assuming that the wave field is weakly non-linear, weakly dispersive, and that these effects are of similar order $\left(U_{r} \sim O(1)\right.$, i.e. Boussinesq regime), the non-linear source term $S_{n l}$ can be approximated with an integral of the bispectrum as follows (Herbers and Burton, 1997; Norheim et al., 1998; Herbers et al., 2000):

$$
S_{n l}(f)=\frac{3 \pi f}{h} \int_{-\infty}^{\infty} \operatorname{Im}\left\{B^{*}\left(f^{\prime}, f-f^{\prime}\right)\right\} d f^{\prime} .
$$

Eq. 11 differs from the expression of Herbers et al. (2000) (their Eq. 2) in several points: the conjugate of $B$ is taken in order to be consistent with their definition of the bispectrum (conjugate of the present definition); $S_{n l}$ is here defined as a function of $f$ (not $\omega$ ) and the definition with the full integral is kept (symmetric properties of the bispectrum are not used to decompose it). Previous studies proposed evolution equations for the energy and bispectra (e.g., the stochastic model of Herbers and Burton, 1997) to simulate the propagation and transformation of directional seas. As in Herbers et al. (2000), we take advantage of the spatial resolution of the present dataset and directly evaluate $S_{n l}$ and $S_{f r}$ at each available cross-shore location using trapezoidal rules for approximating the integrals. Besides removing uncertainties associated with its cross-shore prediction, using measured bispectra has for advantage to relax the limitations of the stochastic Boussinesq model of Herbers and Burton (1997) (such as the distance over which bispectra can be propagated, see Freilich et al., 1984). In order to be consistent with the original equation derived by Herbers and Burton (1997, their Eq. 22a), the energy in Eq. 9 is assumed to propagate at the shallow water wave speed so that $F(f)=\rho g E(f) \sqrt{g h}$, with $\rho$ the water density and $g$ the acceleration of gravity. After these considerations, Eq. 9 simplifies to

$$
\frac{\partial E(f) \sqrt{g h(x)}}{\partial x}=\frac{1}{\rho g}\left(S_{n l}(f)+S_{f r}(f)\right) .
$$

Integrating this equation (in space) between the location of the first gauge $x_{0}$ and any location $x_{1}$ prior to the mean breaking point location yields the following expression for the energy density spectrum at $x_{1}$ $E\left(x_{1}, f\right)$ :

$$
E\left(x_{1}, f\right)=E\left(x_{0}, f\right) \frac{\sqrt{g h\left(x_{0}\right)}}{\sqrt{g h\left(x_{1}\right)}}+\frac{1}{\rho g \sqrt{g h\left(x_{1}\right)}} \int_{x_{0}}^{x_{1}}\left(S_{n l}+S_{f r}\right) d x .
$$


Finally, the ratio $\int_{x_{0}}^{x_{1}} S_{n l} d x / F\left(x_{1}\right)$, which represents the energy flux received (or lost) via non-linear coupling between $x_{0}$ and $x_{1}$ over the total energy flux at $x_{1}$, is used in the following as an approximation of the relative amount of forced energy at $x_{1}$. Outside the surf zone, where dissipative processes dominate, this estimation was found to be more reliable than the bicoherence, which is often used as a proxy for such an estimation but lacks general consensus upon its definition, see Appendix $\mathrm{C}$ for more details.

\section{Wavenumber and phase velocity spectra of shoaling and surf zone waves}

In this section, we present and describe the main results from the cross-spectral and bispectral analyses. The computation of dominant wavenumber $\kappa$ and phase velocity $c$ spectra for varying degrees of nonlinearity resulted in the identification of four different regimes of propagation, which broadly consist of: a linear regime (stage I), a shoaling regime relatively far from the mean breaking point (stage II), a shoaling regime near the mean breaking point (stage III) and a surf zone regime (stage IV). The Ursell number $U_{r}$ (Fig. 2e) was used as a reference to define these regimes of propagation, which are characterized by similar patterns - between all wave tests considered here - in wavenumber and phase velocity spectra. For all stages, $\kappa(f)$ and $c(f)$ spectra are compared to predictions from the linear wave dispersion relation, which links the spatial and temporal information of a linear wave field:

$$
\omega^{2}=g|k| \tanh (|k| h)
$$

In the following, the subscript ' $L$ ' is used throughout the manuscript to refer to $\kappa$ or $c$ values that are solution to Eq. 14 (i.e. $\kappa_{L}$ and $c_{L}=\omega / \kappa_{L}$ ). For conciseness, the propagation as linear waves is discussed using the results from the most non-linear case (A2), already shown in Fig. 3. The other stages of propagation focus on the differences between broad and narrow spectra, i.e. between A2 and A3. Note that $\kappa$ and $c$ characteristics during A1 are very similar to those obtained during A2 for similar $U_{r}$ numbers. Finally, given the relatively high spatial resolution, frequency-wavenumber power spectra $P(\omega, k)(e . g$., see Redor et al., 2019) were computed using 2D Fourier analysis at four different cross-shore sections corresponding to stages I, II, III and IV, by averaging over 63 Hann-windowed time-records of 128 seconds overlapping by $50 \%$ (Fig. 4). The chosen length of the cross-shore section (width of the data window for computing the 2D Fast-Fourier transform) is a best compromise for reaching a proper resolution in wavenumber $k$ and still assuming little change in wave type over the section. The 2D Fourier analysis shown in Fig. 4 provides a qualitative information on energy spreading in $(\omega, k)$ space and is particularly useful to illustrate what the single-valued dominant wavenumber represents in a mixed sea-state. Fig. 4 will be used in the present and following sections to analyse the link between non-linear energy exchanges and the dispersive properties of the wave field.

\subsection{Propagation as free, linear waves (I)}

Fig. 3 shows for the most non-linear case A2 an almost perfect match for $f>0.6 f_{p}$ between the measured wave phases, wavenumbers and phase velocity spectral estimates with the predictions from the linear wave dispersion relation (Fig. 3a, 3c and 3d respectively). The decrease of coherence at high frequencies (Fig. 3b) can be explained by the relatively low energy content in the spectrum tail ( $>86 \%$ of the variance is contained between $0.6 f_{p}$ and $1.5 f_{p}$ during A2). It also provides an explanation for the slight 
deviations of measured $\kappa$ and $c$ from predictions by the linear wave dispersion relation (Fig. 3c-d). However, the 2D Fourier analysis performed over the flat section of the flume (Fig. 4a-b) reveals the presence of energy along the dispersion relation for bound high-frequency components (e.g., see at $f>2.5 f_{p}$ for A2 and around $2 f_{p}$ for A3), suggesting that energy transfers towards high harmonics already occur in this region. Nonetheless, the energy is mainly distributed along the linear dispersion relation, which indicates that short-wave components were mostly propagating as free waves in the deepest regions of the wave flume $\left(U_{r} \sim O(0.01-0.1)\right)$

Data points in the infragravity frequency band $\left(f<0.6 f_{p}\right)$ correspond to wave components generated via difference interactions and that are bound to short-wave groups. As such, bound infragravity waves propagate at a speed close to the corresponding group velocity, which is lower than their intrinsic phase velocity. This is confirmed in Fig. 3d with a good correspondence between measured $c$ values with shortwave groups propagation speed $\left(c_{e n v}\right.$, shown as the orange line), estimated via cross-spectral analysis of short-wave groups envelop. The latter was computed following Janssen et al. (2003), as the absolute value of the analytical signal of $\zeta:|\zeta+i \mathcal{H}\{\zeta\}|$ (no low-pass filtering is needed for the present application). Note that the discrepancies observed at $f \in\left[0.3 f_{p} ; 0.6 f_{p}\right]$ can be explained by the lower levels of energy at these frequencies: $E(f) \sim O\left(10^{-5}\right) \mathrm{m}^{2} / \mathrm{Hz}$ as opposed to $E(f) \sim O\left(10^{-4}\right) \mathrm{m}^{2} / \mathrm{Hz}$ around $f=0.1 f_{p}$.

\subsection{Shoaling of weakly non-linear waves (II)}

At higher Ursell number $\left(U_{r} \sim 0.3\right)$, wave non-linearities become significant and non-linear energy transfers to high harmonics promote the dominance of bound high harmonics (Fig. 4c-d). This is observed in the considerable deviation of dominant wavenumbers and phase velocity spectral estimates from the predictions of the linear dispersion relation for $f>1.5 f_{p}$ (see Fig. 5c-f). During A3, the phase velocity values at $2 f_{p}$ and $3 f_{p}$ are equal to that of the peak frequency (difference $<1 \%, c\left(f_{p}\right)=1.95 \mathrm{~m} / \mathrm{s}$ ), while during A2 they lie between the value at $f_{p}$ and the predictions of the linear dispersion relation $(\sim 7 \%$ difference from $c\left(f_{p}\right)=2.18 \mathrm{~m} / \mathrm{s}$ ). At the second harmonic $2 f_{p}$, most of the non-linear energy transfers occur via sum interactions of components around $f_{p}$ (self-self interactions) as evidenced by the positive imaginary part of $B^{*}$ around $\left(f_{p}, f_{p}\right)$, see Fig. $5 \mathrm{~g}$ and $5 \mathrm{~h}$ for tests A2 and A3 respectively. Although less evident in the bispectra, the normalised bispectra shown in Fig. C1 indicate that strong coupling involving $f_{p}$ and higher harmonics also exist in the shoaling region. These are particularly strong around $\left(2 f_{p}, f_{p}\right)$ for A3, which explains the significant growth of the third harmonic $3 f_{p}$ observed for that test (Fig. 5b). Consistent with field observations (e.g. Elgar and Guza, 1985a; Norheim et al., 1998), relatively strong nonlinear exchanges by sum interactions are observed during A2, despite the broader spectrum conditions (note the scale difference between the A2 and A3 cases). Short-wave frequencies distant from $f_{p}\left(\right.$ e.g. $\left.\sim 1.5 f_{p}\right)$ show a strong coupling with $f_{p}$, which is the consequence of a broader spectrum for A2 compared to A3. A striking result in this regime of propagation is the fact that, despite non-linearities becoming important $\left(\epsilon \sim 0.12-0.2, S_{k} \sim 1\right)$, the principal wave components follow the linear wave dispersion relation. The spatial structure of the wave field for $f \in\left[0.6 f_{p} ; 1.5 f_{p}\right]$ is therefore well-described by the linear wave theory (Fig. $5 \mathrm{c}$ and $5 \mathrm{~d}$ ). However, this is not the case for $f>1.5 f_{p}$, where the overestimation of wavenumbers by the linear dispersion relation increases with $f$ (up to a factor 2.5 at $3.5 f_{p}$ ). As the biphase at both $\left(f_{p}, f_{p}\right)$ and $\left(f_{p}, 2 f_{p}\right)$ are close to 0 , this regime of propagation is consistent with Stokes-like non-linearities (e.g., 
see Elgar and Guza, 1985a).

In the infragravity band, wave components are still bound to short-wave groups as indicated by the good match between spectral estimates of phase velocity and the propagation speed of short-wave groups. Energy transfers towards the infragravity band concentrate at frequencies around 0.1-0.2 $f_{p}$ (Fig. 5a and $5 \mathrm{~b})$ and principally originate from strong difference interactions which transfer energy from $f_{p}$ towards that infragravity frequency and components at frequency slightly lower than $f_{p}$ (see the negative imaginary part of $B^{*}$ along the $f_{p}$ anti-diagonal, Fig. $5 \mathrm{~g}$ and $\left.5 \mathrm{~h}\right)$.

\subsection{Shoaling of non-linear waves in the vicinity of the breaking point (III)}

The next regime analysed in Fig. 6 corresponds to non-linear waves approaching the mean breaking point location $\left(U_{r} \sim 0.7\right)$. At this Ursell number, wavenumber and phase velocity spectra become less frequency-dependent at high frequencies. This can be explained by the fact that most of the energy at those frequencies is bound to primary components in the range $\left[0.6 f_{p} ; 1.5 f_{p}\right]$, and that $c$ values display little variations at these frequencies $\left(c / c_{L}\left(f_{p}\right)\right.$ is within $5 \%$ of $\left.c\left(f_{p}\right) / c_{L}\left(f_{p}\right)\right)$. As a result, wavenumbers at high frequencies are relatively well described by a simple dispersion relation given by $\kappa(f)=2 \pi f / c\left(f_{p}\right)$ (Fig. 6c-d). Note that at these depths ( $\kappa_{p} h \sim 0.57$ and 0.47 for A2 and A3 respectively), this approximately corresponds to the shallow water dispersion relation $\kappa_{s w}(f)=2 \pi f / \sqrt{g h}$, which explains why most of the energy is spread around this dispersion relation in Fig 4e-f. Non-linear amplitude effects become increasingly important $\left(\epsilon=0.25\right.$ and $\left.S_{k}=1.4-1.5\right)$ and deviations between $c\left(f_{p}\right)$ and predictions by the linear dispersion relation are observed (Fig. 6e-f). As noted and observed by Herbers et al. (2002), this is the result of nonlinear interactions, which alter the wavenumber of all three wave components involved. Indeed, bound high harmonics contribute to an increase in skewness and height of principal wave crests, which has for effect to enhance their propagation speed. Variations in the strength of non-linear interactions thus explain the varying magnitude of the deviations of $c\left(f_{p}\right)$ from predictions by the linear dispersion relation as observed in both field and laboratory settings (Thornton and Guza, 1982; Herbers et al., 2002; Tissier et al., 2011). For this range of Ursell number, overestimations of $\kappa$ by the linear dispersion relation remain close to a factor 2 at $f=3.5 f_{p}$.

In these regions of the wave flume, surface elevation bispectra are still dominated by real values (note the x2 in imaginary values, Fig. 6g-h), typical of (horizontally) skewed and nearly symmetric waves $(A s \sim 0$, see Fig. 2c-d and also Masuda and Kuo, 1981; Elgar and Guza, 1985a). The strong coupling between first and second harmonics, which for instance explains the energy peak at $3 f_{p}$ in A3 (Fig. 6b) is here evident in the imaginary part of the bispectra at $\left(f_{p}, 2 f_{p}\right)$ (see also the bicoherence in Fig. C1). Infragravity frequencies between 0.1-0.2 $f_{p}$ keep receiving energy via relatively strong difference interactions. As a consequence, prior to short-wave breaking infragravity waves are still bound to short-wave groups during both tests (see Fig. 6e-f, and also de Bakker et al., 2015).

\subsection{Surf zone waves (IV)}

In the surf zone $\left(U_{r}\right.$ taken at 3.2), wavenumber and phase velocity spectra are frequency-independent (Fig. 7e-f). The large differences observed between the measured phase velocity at peak frequency and that predicted with the linear dispersion relation demonstrate the dominance of amplitude effects over dispersive 
ones (Herbers et al., 2002). All components travel at a speed slightly larger than that of shallow water waves ( $\sim 10-20 \%$ depending on the wave test), which is consistent with many past field and laboratory observations of wave dispersion in the surf zone (Inman et al., 1971; Svendsen et al., 1978; Stive, 1980; Thornton and Guza, 1982, and many others). For both A2 and A3 tests, the relation $c=\sqrt{g h(1+\epsilon)}$ is shown in Fig. 7e-f (see also Fig 4g-h, for the corresponding frequency-wavenumber surface elevation power spectra). This formula is asymptotic in shallow water to the non-linear dispersion relation given by Hedges (1976) with the adjustment of Booij (1981) (see also Catalán and Haller, 2008, for an assessment of these relations for depth-inversion applications). In the inner surf zone, where $\epsilon \sim 0.3$ during all tests (Fig. 2b), this yields $c \sim 1.14 \sqrt{g h}$, a relation also found in the field by Tissier et al. (2011). This relation appears as an upper bound, in an average sense (e.g. over several wave groups), for the propagation speed of wave components in the inner surf zone.

In the short-wave frequency range, bispectra display relatively weak and nearly equal real and imaginary parts, characteristics of asymmetric (pitched forward) broken waves, with biphases approaching $-\pi / 2$ (Elgar and Guza, 1985a; Doering and Bowen, 1987): for instance during A3, $\beta\left(f_{p}, f_{p}\right) \sim \beta\left(f_{p}, 2 f_{p}\right) \sim-\pi / 3$. Since these values were still close to 0 during stage III, this indicates that bound high-harmonic components (here only shown for $2 f_{p}$ and $3 f_{p}$ ) slowly drift out of phase from the principal component starting from the mean breaking point location. This is in contrast with short-wave group-forced bound infragravity waves that slowly drift out of phase with the short-wave envelope in the shoaling region (e.g., see Elgar and Guza, 1985a). Since this process occurs over long distances, the differences in phase velocities between $f_{p}, 2 f_{p}$ and $3 f_{p}$ are not sufficiently large to be observed in the present dataset. Finally, in the inner surf zone, intense and complex energy transfers occur within the infragravity band and also between infragravity and short-wave components. For more information on these processes during the GLOBEX experiments, the reader is referred to the study of de Bakker et al. (2015).

\section{Role of non-linear energy transfers on $\kappa$ and $c$}

Most differences observed in wavenumber and phase velocity spectra between broad and narrow-banded wave conditions concentrate in the shoaling region (stages II and III). In stage II, $c$ values at $2 f_{p}$ and $3 f_{p}$ during A2 lie between $c\left(f_{p}\right)$ and the values predicted by linear wave theory while $c\left(f_{p}\right), c\left(2 f_{p}\right)$ and $c\left(3 f_{p}\right)$ are all equal during A3 (Fig. 5e-f). As these differences between broad versus narrow-band spectra are likely explained by the relative importance of forced energy at those frequencies (see the cross-spectral analysis performed on synthetic data and discussed in Appendix A and also Herbers and Guza, 1992; Herbers et al., 2000), we analyse non-linear energy transfers to $2 f_{p}$ and $3 f_{p}$ in more details here.

Wave amplitudes at $f_{p}, 2 f_{p}$ and $3 f_{p}\left(a_{f_{p}}, a_{2 f_{p}}\right.$ and $a_{3 f_{p}}$ respectively) computed from energy spectra modelled with Eq. 13 are compared against observations in Fig. 8. Overall, the Boussinesq approach of Herbers and Burton (1997) for the non-linear energy transfers between triads accurately predicts the growth of second and third harmonics across the shoaling zone for both broad and narrow-band wave tests. The cross-shore evolution of $a_{f_{p}}, a_{2 f_{p}}$ and $a_{3 f_{p}}$ are very well described up to the mean breaking point, with mean absolute percentage errors (MAPE) lower than $5 \%$ and $13 \%$ for $a_{2 f_{p}}$ and $a_{3 f_{p}}$ respectively. With respect to the amount of (free) energy imposed at the paddle, the narrow-banded conditions during A3 
promote more efficient energy transfers, with growths of $a_{2 f_{p}}$ and $a_{3 f_{p}}$ by a factor 3 and 10 respectively, while these factors are only between 1.5 and 2 during A2.

A closer look at the cross-shore evolution of the source terms (Fig. 9) shows that friction effects are negligible at high frequencies but not around the peak frequency where the energy dissipated through bottom friction is of similar order than the energy lost via non-linear coupling. During A3 (Fig. 9b), the steady increase of $S_{n l}\left(2 f_{p}\right)$ indicates a gradual growth of $a_{2 f_{p}}$ in the shoaling region (see also Fig. 8b). This growth is most intense right before the mean breaking point location, around the location corresponding to stage III. Computations of the ratio $\int_{x_{0}}^{x_{1}} S_{n l} d x / F\left(x_{1}\right)$ at the location $x_{1}$ corresponding to stage II (resp. III) suggest that approximately $70 \%$ (resp. 80\%) of the energy at $2 f_{p}$ is forced during A3. At $3 f_{p}$, these estimations range between $90 \%$ and $100 \%$ during stage II and III respectively. Although the resolution in wavenumbers does not allow a clear separation of forced and free energy at $2 f_{p}$, the frequency-wavenumber power spectra shown in Fig. 4d (II) and 4f (III) corroborate these numbers and clearly indicate that most of the energy at $3 f_{p}$ is forced. In contrast, non-linear energy exchanges between triads display a more complex picture for the broader conditions of A2. $S_{n l}\left(2 f_{p}\right)$ oscillates around 0 so that when spatially integrated, non-linear energy exchanges explain only $20 \%(30 \%)$ and $30 \%(50 \%)$ of the total energy at $2 f_{p}$ and $3 f_{p}$ respectively during stage II (III). These numbers are quite consistent with the bicoherence values shown in Appendix C. For both wave tests, $b^{2}$ values are found to vary little between stage II and III: for A2, $b^{2}\left(f_{p}, f_{p}\right) \sim 0.3$ and $b^{2}\left(f_{p}, 2 f_{p}\right) \sim 0.2$, while these values oscillate between 0.55 and 0.65 for A3. $b^{2}\left(f_{p}, f_{p}\right)$ and $b^{2}\left(f_{p}, 2 f_{p}\right)$ can be considered as crude estimates of the relative amount of forced energy at $2 f_{p}$ and $3 f_{p}$ respectively (Kim and Powers, 1979).

The effect of varying relative amounts of forced energy not only explain the differences observed in $\kappa$ and $c$ spectra at high frequencies between A2 and A3 but also their variation across frequencies. During A3 for instance, non-linear energy transfers in the short-wave frequency band were predominantly towards $2 f_{p}$ and $3 f_{p}$ (Fig. $5 \mathrm{~h}$ and $6 \mathrm{~h}$ ). At these harmonics, energy is predominantly forced ( $>70 \%$ around $2 f_{p}$ and $>90 \%$ around $3 f_{p}$ ), thus, the dominant $\kappa$ and corresponding $c$ values are directly related to the values at the peak frequency. In contrast, frequencies located in valleys between harmonics (i.e. $1.5 f_{p}$ and $2.5 f_{p}$ ) receive very little energy through the coupling of triads. This eventually leads to the patterns in $c$ observed in Fig. 5f, which are very similar to those obtained by Crawford et al. (1981) with their model for modulated wave trains. During A2, lower relative amounts of forced energy are found at high harmonics and dominant wavenumbers lie between $\kappa\left(f_{p}\right)$ and the intrinsic value at that frequency predicted by the linear dispersion relation. Furthermore, due to the broader spectrum conditions, this forced energy is more spread across frequencies, which explains the weaker variations of $\kappa$ and $c$ across frequencies as compared to A3 (e.g., see Fig. 5e-f).

The present study considers unidirectional wave fields transforming across a mildly sloping beach. A typical field situation where incident wave spectra exhibit varying degrees of directional spreading is unclear as far as non-linear energy transfers are concerned. According to Boussinesq theory (e.g., Herbers and Burton, 1997), directional spreading has only a weak influence on the efficiency of non-linear energy transfers by sum interactions, as opposed to difference interactions. For instance, these authors predicted a weaker growth of high-harmonic bound wave by $10-20 \%$ for very large directional spreads $\left(60^{\circ}\right)$. For more realistic 
spreading angles in the nearshore, de Wit et al. (2020) also recently noted variations of approximately $10 \%$. Although small, such a decrease in efficiency of non-linear energy transfer towards high harmonics due to an increasing directional spreading might lead to lower relative amounts of forced energy, subsequently leading to slightly less important deviations of $\kappa$ and $c$ spectral estimates from predictions by the linear wave dispersion relation (see also Appendix A). The larger spectral bandwidth generally associated with wave spectra exhibiting a large directional spreading is, however, likely to be the principal reason explaining weaker couplings between triads and thus weaker relative amounts of forced energy at high harmonics.

\section{Discussion and concluding remarks}

Cross-spectral and bispectral analyses were employed on a highly-resolved surface elevation dataset to study the dispersive properties of waves shoaling and breaking over a mildly sloping beach. For all wave tests considered here, four regimes of propagation (I to IV) with specific characteristics in dominant wavenumber and phase velocity spectra could be defined using a local Ursell number. Stage II $\left(U_{r} \sim 0.3\right)$ is particularly interesting as it simultaneously shows significant non-linear effects at high harmonics (typically $f>1.5 f_{p}$ ), which are evidenced by the large deviations of $\kappa$ and $c$ spectra from predictions by the linear wave dispersion relation, and a clearly linear spatial field for the primary components. Although less energy was imposed during A3 as compared to A2, the narrow-banded conditions promoted relatively more important non-linear energy transfers towards high harmonics such that phase velocity at $2 f_{p}$ and $3 f_{p}$ were found equal to those at the peak frequency. For A2, with a broader spectrum imposed, forced energy at high frequencies was found in relatively smaller proportion and $c$ spectral estimates lied between values at the peak frequency and that given by the linear dispersion relation. Closer to the mean breaking point (stage III), non-linear energy transfers were found more intense, which, together with the less dispersive conditions compared to stage II explain that $\kappa$ and $c$ spectra become less frequency dependent. In the surf zone (stage IV), wavenumber and phase velocity spectra essentially showed the same characteristics for all tests and a modified shallow water dispersion that accounts for non-linearity $(c=\sqrt{g h(1+\epsilon)})$ accurately describes the dispersive properties of the wave field. Some of the results presented here for shoaling and breaking waves confirm previous findings obtained in the field (Thornton and Guza, 1982; Elgar and Guza, 1985b; Herbers et al., 2002, among others), yet the unprecedented level of accuracy obtained with the GLOBEX dataset allowed to highlight and quantify the effect of varying levels of forced energy at high harmonics on the dispersive properties of the wave field.

The detailed analysis of the different regimes of propagation highlighted here indirectly provides an assessment of the validity of the linear wave dispersion relation at several locations in the nearshore and for a range of wave conditions. Two specific regions in the short-wave frequency band can be defined: primary components $\left(f \in\left[0.6 f_{p}, 1.5 f_{p}\right]\right)$ and high harmonics $\left(f>1.5 f_{p}\right)$. For the primary components, deviations from the linear wave dispersion relation are due to non-linear amplitude effects and concentrate in a region near the mean breaking point and in the surf zone. These effects can cause deviations of $\kappa$ and $c$ from predictions by the linear wave dispersion of the order of $10-20 \%$. At high frequencies, deviations are much larger $(O(100 \%))$ and also occur much farther seaward. For instance, stage II corresponds to $\kappa_{p} h=0.71$, 0.70 and 0.62 for wave tests A1, A2 and A3 respectively. The fact that such deviations from predictions 
by the linear dispersion relation are found in $\kappa$ and $c$ spectra so far seaward of the surf zone for different wave conditions is expected to have strong implications for a wide range of applications, two of which are briefly discussed below.

Depth-inversion algorithms such as cBathy (Holman et al., 2013) estimate the water depth by applying the linear wave dispersion relation to the most coherent pairs of wave frequency and wavenumber extracted from timeseries of video images (Stockdon and Holman, 2000; Plant et al., 2008). However, due to the working principles of video cameras, video data can be more coherent at high harmonics and frequencies greater than $1.5 f_{p}$ are often picked to invert the water depth (Stockdon and Holman, 2000; Bergsma and Almar, 2018). In such cases, large deviations of the dominant wave phase velocity from linear wave theory are expected, which likely explains why Brodie et al. (2018) noted an overestimation in the detected phase velocities by $20-100 \%$ up to $50 \mathrm{~m}$ seaward of the mean breaking point location. In the absence of knowledge on the relative amounts of forced energy at high harmonics, pairs of frequencies and wavenumbers around the peak frequency should be preferred in regions where non-linearities are expected. Similarly, the large overestimation of $\kappa$ by the linear dispersion relation causes the widely reported blow-up at high harmonics when correcting sub-surface pressure signals for depth attenuation (Bonneton and Lannes, 2017; Mouragues et al., 2019; Martins et al., 2020b). The use of a cutoff frequency prevents for instance the accurate description of third-order parameters and wave height distributions (e.g., see Martins et al., 2020a). In shallow water $(\mu \lesssim 0.3)$, a weakly dispersive formula recently developed allows for an accurate correction of the energy levels at high harmonics both seaward of (Bonneton et al., 2018; Mouragues et al., 2019) and in the surf zone (Martins et al., 2020b). However in deeper water, a non-linear fully dispersive reconstruction requires knowledge on the spatial structure of the wave field, which is generally lacking.

\section{Acknowledgments}

Kévin Martins greatly acknowledges the financial support from the University of Bordeaux, through an International Postdoctoral Grant (Idex, nb. 1024R-5030). The GLOBEX project was supported by the European Community's Seventh Framework Programme through the Hydralab IV project, EC Contract 261520. The GLOBEX data used in this research can be accessed on Zenodo at https://zenodo.org/record/4009405 or from the authors, and can be used under the Creative Commons Attribution 4.0 International licence. The bispectral analysis tools developed and used in this study are accessible from the first author github repository at https://github.com/ke-martins/bispectral-analysis. Fruitful exchanges with Steve Elgar regarding the bispectral analysis were greatly appreciated. The authors thank two anonymous reviewers for their helpful comments and suggestions.

\section{Appendix A. Sensitivity analysis of the cross-spectral technique to the presence of forced energy}

The situation where both free and forced components exist in a wave field is not entirely clear as far as the cross-spectral analysis is concerned. Some studies consider the cross-spectral estimates to be biased towards bound harmonics (e.g., see Lake and Yuen, 1978; Thornton and Guza, 1982), arguing that the travelling distance between two gauges is reduced for bound components compared to free ones. This is 
evidently exacerbated when using sub-surface pressure sensors or current meters since bound harmonics are much less attenuated and will dominate the spectrum at some depth.

To analyse the sensitivity of the cross-spectral analysis to different relative levels of forced energy, some tests are performed here on synthetic data. Starting at a position $x_{1}$, a surface elevation timeseries following a JONSWAP spectrum with similar parameters to the A3 test is generated (Fig. A1). A Gaussian-shaped perturbation around $2 f_{p}$ is added to represent forced harmonics: for any $f$ around $2 f_{p}$, the forced harmonic wavenumber is set at $\kappa_{\text {forced }}=2 \kappa(f / 2)$, so that we have $c_{\text {forced }}(f)=c(f / 2)$. For the phases of forced harmonics, we choose to impose $\theta_{\text {forced }}(f)=\theta(f / 2)$ for simplicity. The maximum ratio of forced to free harmonic amplitudes $a_{2 f_{p}, \text { forced }} / a_{2 f_{p}, \text { free }}$ is set to 6 , which corresponds to the maximum ratio of energy $E\left(f_{p}\right) / E\left(2 f_{p}\right)$ of approximately 0.4 that was observed in the flume outside the surf zone. Each wave component is then propagated using its corresponding wavenumber to a second location $x_{2}$.

The results from the cross-spectral analysis performed on the synthetic timeseries at $x_{1}$ and $x_{2}$ are provided in Fig. A2 as the wavenumber and phase speed at $2 f_{p}$ shown as a function of $a_{2 f_{p}, \text { forced }} / a_{2 f_{p}, \text { free }}$. In the absence of forced components $\left(a_{2 f_{p}, \text { forced }} / a_{2 f_{p}, \text { free }}=0\right)$, components around $2 f_{p}$ follow the dispersion relation given by linear wave theory, i.e. $\kappa\left(2 f_{p}\right)=\kappa_{L}\left(2 f_{p}\right)$ and $c\left(2 f_{p}\right)=c_{L}\left(2 f_{p}\right)$. Interestingly, when forced and free components are found in equal proportion $\left(a_{2 f_{p}}\right.$,forced $/ a_{2 f_{p}}$,free $\left.=1\right)$, the relation $\kappa\left(2 f_{p}\right) \sim\left(\kappa_{L}\left(2 f_{p}\right)+2 \kappa_{L}\left(f_{p}\right)\right) / 2$ is verified. For higher ratio of forced to free energy, $\kappa\left(2 f_{p}\right)$ and $c\left(2 f_{p}\right)$ values rapidly converge towards the values corresponding to the peak frequency. Overall, these results suggest that the cross-spectral analysis is not biased towards forced components, but simply provides a dominant wavenumber (or averaged in terms of energy) and its corresponding phase velocity.

\section{Appendix B. Validation of the bispectrum computations using the skewness and asymmetry test}

The wave skewness and asymmetry are third-order moments characterizing the wave shape. These parameters are directly related to the energy content at high frequencies of the spectra and bispectra (Elgar, 1987). The fact that the statistical (see Eq. 1 and 2) and the bispectrum-based definitions are theoretically equivalent provide a means to validate the bispectrum's calculations.

Due to its symmetry properties, the bispectrum can be uniquely defined in a single octant in the frequency-space (Hasselmann et al., 1963; Elgar and Guza, 1985a). If we denote by $f_{N}$ the Nyquist frequency, the octant with positive frequencies is bounded by the vertices at $(0,0),\left(f_{N / 2}, f_{N / 2}\right)$ and $\left(f_{N}, 0\right)$. The wave skewness and asymmetry are defined by the sum of the real and imaginary parts of the bispectrum over this octant respectively, normalized by the cube of the free surface elevation standard deviation (Elgar, 1987; Elgar and Guza, 1985a). This reads:

$$
\begin{aligned}
& S_{k}=\left[12 \sum_{n} \sum_{l} \operatorname{Re}\left\{B\left(f_{n}, f_{l}\right)\right\}+6 \sum_{n} \operatorname{Re}\left\{B\left(f_{n}, f_{n}\right)\right\}\right] /{\overline{(\zeta-\bar{\zeta})^{2}}}^{3 / 2} \\
& A_{s}=\left[12 \sum_{n} \sum_{l} \operatorname{Im}\left\{B\left(f_{n}, f_{l}\right)\right\}+6 \sum_{n} \operatorname{Im}\left\{B\left(f_{n}, f_{n}\right)\right\}\right] /{\overline{(\zeta-\bar{\zeta})^{2}}}^{3 / 2}
\end{aligned}
$$

with $n>l$ and $n+l<N$.

The cross-comparison between statistical and bispectrum-based definitions of the wave skewness and asymmetry computed for A3 is provided in Fig. B1 and shows a perfect match between both definitions, 
thus validating the present computations of the bispectrum. Note that for these comparisons, blocks were not tapered and rectangular windows were used for computing the Fast Fourier Transforms. Using any other types of windows resulted in small differences between the two definitions of both third-order wave parameters.

\section{Appendix C. Normalised bispectra (bicoherence)}

We generally seek a normalisation of the bispectrum so that it takes 1 as value when there is a full coupling between the three components involved and 0 when there is none. In the case of surface gravity waves, this would correspond to a situation with the only presence of forced or free energy respectively. At present, there does not seem to be a consensus in the literature on what definition of the bicoherence should be used to robustly quantify or at least characterize the relative dominance of forced energy (e.g. Haubrich, 1965; Kim and Powers, 1979; Elgar and Guza, 1985a; Hinich and Wolinsky, 2005; de Bakker et al., 2015, and many others). The normalisation proposed by Haubrich (1965) is frequently used for studying non-linearities in the nearshore area but was shown to lead to values greater than 2 (e.g. Elgar and Guza, 1985a). In the present study, this was also the case, but only when merging across frequencies was performed beforehand. Nonetheless, a slightly different normalisation that was proposed in Hagihira et al. (2001) is used in the present case as it seems the most appropriate normalisation (see their Appendix):

$$
b\left(f_{1}, f_{2}\right)=\frac{\left|B\left(f_{1}, f_{2}\right)\right|}{\mathcal{E}\left[\left|A\left(f_{1}\right)\right| \cdot\left|A\left(f_{2}\right)\right| \cdot\left|A^{*}\left(f_{1}+f_{2}\right)\right|\right]}
$$

As explained in Hagihira et al. (2001), the bicoherence is 1 only when phase angles of all triple products are equal.

Squared bicoherence values $b^{2}$ for both II and III regimes of propagation are shown in Fig. C1. As frequency merging is inappropriate with this definition of the bicoherence (due to the expected value in the denominator), $b$ values were averaged over a $3 \times 3$ square in the frequency space.

\section{References}

de Bakker, A.T.M., Herbers, T.H.C., Smit, P.B., Tissier, M.F.S., Ruessink, B.G., $2015 . \quad$ Nonlinear infragravity-wave interactions on a gently sloping laboratory beach. Journal of Physical Oceanography 45, 589 -605. doi:10.1175/JPO-D-14-0186.1.

Bergsma, E.W.J., Almar, R., 2018. Video-based depth inversion techniques, a method comparison with synthetic cases. Coastal Engineering 138, 199 - 209. doi:10.1016/j.coastaleng.2018.04.025.

Berni, C., Barthélemy, E., Michallet, H., 2013. Surf zone cross-shore boundary layer velocity asymmetry and skewness: An experimental study on a mobile bed. Journal of Geophysical Research: Oceans 118, 2188 - 2200. doi:10.1002/jgrc.20125.

Bonneton, P., Bruneau, N., Castelle, B., Marche, F., 2010. Large-scale vorticity generation due to dissipating waves in the surf zone. Discrete \& Continuous Dynamical Systems - B 13, 729 -738. doi:10.3934/dcdsb.2010.13.729. 
Bonneton, P., Lannes, D., 2017. Recovering water wave elevation from pressure measurements. Journal of Fluid Mechanics 833, 399-429. doi:10.1017/jfm.2017.666.

Bonneton, P., Lannes, D., Martins, K., Michallet, H., 2018. A nonlinear weakly dispersive method for recovering the elevation of irrotational surface waves from pressure measurements. Coastal Engineering 138, 1 -8. doi:10.1016/j.coastaleng.2018.04.005.

Booij, N., 1981. Gravity waves on water with non-uniform depth and current. Ph.D. thesis. Technische Hogeschool, Delft (Netherlands).

Brodie, K.L., Palmsten, M.L., Hesser, T.J., Dickhudt, P.J., Raubenheimer, B., Ladner, H., Elgar, S., 2018. Evaluation of video-based linear depth inversion performance and applications using altimeters and hydrographic surveys in a wide range of environmental conditions. Coastal Engineering 136, 147 160. doi:10.1016/j.coastaleng.2018.01.003.

Bühler, O., Jacobson, T.E., 2001. Wave-driven currents and vortex dynamics on barred beaches. Journal of Fluid Mechanics 449, 313-339. doi:10.1017/S0022112001006322.

Castelle, B., Scott, T., Brander, R.W., McCarroll, R.J., 2016. Rip current types, circulation and hazard. Earth-Science Reviews 163, 1-21. doi:10.1016/j.earscirev.2016.09.008.

Catalán, P.A., Haller, M.C., 2008. Remote sensing of breaking wave phase speeds with application to non-linear depth inversions. Coastal Engineering 55, 93-111. doi:10.1016/j.coastaleng.2007.09.010.

Clark, D.B., Elgar, S., Raubenheimer, B., 2012. Vorticity generation by short-crested wave breaking. Geophysical Research Letters 39. doi:10.1029/2012GL054034.

Coantic, M., Ramamonjiarisoa, A., Mestayer, P., Resch, F., Favre, A., 1981. Wind-water tunnel simulation of small-scale ocean-atmosphere interactions. Journal of Geophysical Research: Oceans 86, 6607-6626. doi:10.1029/JC086iC07p06607.

Crawford, D.R., Lake, B.M., Yuen, H.C., 1981. Effects of nonlinearity and spectral bandwidth on the dispersion relation and component phase speeds of surface gravity waves. Journal of Fluid Mechanics 112, 1 -32. doi:10.1017/S0022112081000281.

Doering, J.C., Bowen, A.J., 1986. Shoaling surface gravity waves: A bispectral analysis, in: Proceedings of the 20th Conference on Coastal Engineering, Taipei, Taiwan, pp. 150-162. doi:10.1061/9780872626003.012.

Doering, J.C., Bowen, A.J., 1987. Skewness in the nearshore zone: A comparison of estimates from marshmcbirney current meters and colocated pressure sensors. Journal of Geophysical Research: Oceans 92, 13173 - 13183. doi:10.1029/JC092iC12p13173.

Doering, J.C., Bowen, A.J., 1995. Parametrization of orbital velocity asymmetries of shoaling and breaking waves using bispectral analysis. Coastal Engineering 26, 15-33. doi:10.1016/0378-3839(95)00007-X. 
Donelan, M.A., Hamilton, J., Hui, W.H., Stewart, R.W., 1985. Directional spectra of wind-generated ocean waves. Philosophical Transactions of the Royal Society of London. Series A, Mathematical and Physical Sciences 315, 509-562. doi:10.1098/rsta.1985.0054.

Drazen, D.A., Melville, W.K., 2009. Turbulence and mixing in unsteady breaking surface waves. Journal of Fluid Mechanics 628, 85-119. doi:10.1017/S0022112009006120.

Elgar, S., 1987. Relationships involving third moments and bispectra of a harmonic process. IEEE Transactions on Acoustics, Speech, and Signal Processing 35, 1725-1726. doi:10.1109/TASSP.1987.1165090.

Elgar, S., Freilich, M.H., Guza, R.T., 1990. Model-data comparisons of moments of nonbreaking shoaling surface gravity waves. Journal of Geophysical Research: Oceans 95, 16055-16063. doi:10.1029/JC095iC09p16055.

Elgar, S., Guza, R.T., 1985a. Observations of bispectra of shoaling surface gravity waves. Journal of Fluid Mechanics 161, 425-448. doi:10.1017/S0022112085003007.

Elgar, S., Guza, R.T., 1985b. Shoaling gravity waves: comparisons between field observations, linear theory, and a nonlinear model. Journal of Fluid Mechanics 158, 47-70. doi:10.1017/S0022112085002543.

Freilich, M.H., Guza, R.T., Whitham, G.B., 1984. Nonlinear effects on shoaling surface gravity waves. Philosophical Transactions of the Royal Society of London. Series A, Mathematical and Physical Sciences 311, 1 -41. doi:10.1098/rsta.1984.0019.

Guza, R.T., Thornton, E.B., 1980. Local and shoaled comparisons of sea surface elevations, pressures, and velocities. Journal of Geophysical Research: Oceans 85, 1524-1530. doi:10.1029/JC085iC03p01524.

Hagihira, S., Takashina, M., Mori, T., Mashimo, T., Yoshiya, I., 2001. Practical issues in bispectral analysis of electroencephalographic signals. Anesthesia \& Analgesia 93, 966 - 970. doi:10.1097/00000539200110000-00032.

Hasselmann, K., 1962. On the non-linear energy transfer in a gravity-wave spectrum. Part 1. General theory. Journal of Fluid Mechanics 12, 481-500. doi:10.1017/S0022112062000373.

Hasselmann, K., Munk, W., MacDonald, G., 1963. Bispectra of ocean waves, in: Proceedings of the Symposium on Time Series Analysis, M. Rosenblatt, Ed., New York: Wiley. pp. 125-139.

Haubrich, R.A., 1965. Earth noise, 5 to 500 millicycles per second: 1. spectral stationarity, normality, and nonlinearity. Journal of Geophysical Research (1896-1977) 70, 1415-1427. doi:10.1029/JZ070i006p01415.

Hedges, T.S., 1976. An empirical modification to linear wave theory. Proceedings of the Institution of Civil Engineers 61, 575-579. doi:10.1680/iicep.1976.3408.

Herbers, T.H.C., Burton, M.C., 1997. Nonlinear shoaling of directionally spread waves on a beach. Journal of Geophysical Research: Oceans 102, 21101-21114. doi:10.1029/97JC01581. 
Herbers, T.H.C., Elgar, S., Guza, R.T., 1994. Infragravity-frequency (0.005-0.05 Hz) motions on the shelf. Part I: Forced waves. Journal of Physical Oceanography 24, 917-927. doi:10.1175/15200485(1994)024;0917:IFHMOT¿2.0.CO;2.

Herbers, T.H.C., Elgar, S., Sarap, N.A., Guza, R.T., 2002. Nonlinear dispersion of surface gravity waves in shallow water. Journal of Physical Oceanography 32, 1181-1193. doi:10.1175/15200485(2002)032¡1181:NDOSGW ¿2.0.CO;2.

Herbers, T.H.C., Guza, R.T., 1991. Wind-wave nonlinearity observed at the sea floor. Part I: Forced-wave energy. Journal of Physical Oceanography 21, 1740-1761. doi:10.1175/15200485(1991)021¡1740:WWNOAT ¿2.0.CO;2.

Herbers, T.H.C., Guza, R.T., 1992. Wind-wave nonlinearity observed at the sea floor. Part II: Wavenumbers and third-order statistics. Journal of Physical Oceanography 22, 489-504. doi:10.1175/15200485(1992)022;0489:WWNOAT ¿2.0.CO;2.

Herbers, T.H.C., Guza, R.T., 1994. Nonlinear wave interactions and high-frequency seafloor pressure. Journal of Geophysical Research: Oceans 99, 10035-10048. doi:10.1029/94JC00054.

Herbers, T.H.C., Lowe, R.L., Guza, R.T., 1992. Field observations of orbital velocities and pressure in weakly nonlinear surface gravity waves. Journal of Fluid Mechanics 245, 413-435. doi:10.1017/S0022112092000521.

Herbers, T.H.C., Russnogle, N.R., Elgar, S., 2000. Spectral energy balance of breaking waves within the surf zone. Journal of Physical Oceanography 30, 2723-2737. doi:10.1175/15200485(2000)030ز2723:SEBOBW ¿2.0.CO;2.

Hinich, M.J., Wolinsky, M., 2005. Normalizing bispectra. Journal of Statistical Planning and Inference 130, 405-411. doi:10.1016/j.jspi.2003.12.022.

Hoefel, F., Elgar, S., 2003. Wave-induced sediment transport and sandbar migration. Science 299, 18851887. doi:10.1126/science.1081448.

Holland, T.K., 2001. Application of the linear dispersion relation with respect to depth inversion and remotely sensed imagery. IEEE Transactions on Geoscience and Remote Sensing 39, 2060-2072. doi:10.1109/36.951097.

Holman, R., Plant, N., Holland, T., 2013. cBathy: A robust algorithm for estimating nearshore bathymetry. Journal of Geophysical Research: Oceans 118, 2595-2609. doi:10.1002/jgrc.20199.

Inman, D.L., Tait, R.J., Nordstrom, C.E., 1971. Mixing in the surf zone. Journal of Geophysical Research (1896-1977) 76, 3493-3514. doi:10.1029/JC076i015p03493.

Janssen, T.T., Battjes, J.A., van Dongeren, A.R., 2003. Long waves induced by short-wave groups over a sloping bottom. Journal of Geophysical Research: Oceans 108. doi:10.1029/2002JC001515. 
Kim, Y.C., Beall, J.M., Powers, E.J., Miksad, R.W., 1980. Bispectrum and nonlinear wave coupling. The Physics of Fluids 23, 258-263. doi:10.1063/1.862966.

Kim, Y.C., Powers, E.J., 1979. Digital Bispectral Analysis and Its Applications to Nonlinear Wave Interactions. IEEE Transactions on Plasma Science 7, 120-131. doi:10.1109/TPS.1979.4317207.

Laing, A.K., 1986. Nonlinear properties of random gravity waves in water of finite depth. Journal of Physical Oceanography 16, 2013-2030. doi:10.1175/1520-0485(1986)016¡2013:NPORGW ¿2.0.CO;2.

Lake, B.M., Yuen, H.C., 1978. A new model for nonlinear wind waves. Part 1. Physical model and experimental evidence. Journal of Fluid Mechanics 88, 33-62. doi:10.1017/S0022112078001974.

Longuet-Higgins, M.S., 1956. The refraction of sea waves in shallow water. Journal of Fluid Mechanics 1, 163 - 176. doi:10.1017/S0022112056000111.

Longuet-Higgins, M.S., Stewart, R.W., 1962. Radiation stress and mass transport in gravity waves, with application to 'surf beats'. Journal of Fluid Mechanics 13, 481-504. doi:10.1017/S0022112062000877.

Longuet-Higgins, M.S., Stewart, R.W., 1964. Radiation stresses in water waves; a physical discussion, with applications. Deep Sea Research and Oceanographic Abstracts 11, 529-562. doi:10.1016/00117471(64)90001-4.

Martins, K., Bonneton, P., Bayle, P.M., Blenkinsopp, C.E., Mouragues, A., Michallet, H., 2020a. Surf zone wave measurements from lidar scanners: Analysis of non-hydrostatic processes. Journal of Coastal Research 95, 1189-1194. doi:10.2112/SI95-231.1.

Martins, K., Bonneton, P., Mouragues, A., Castelle, B., 2020b. Non-hydrostatic, non-linear processes in the surf zone. Journal of Geophysical Research: Oceans 125, e2019JC015521. doi:10.1029/2019JC015521.

Masuda, A., Kuo, Y.Y., 1981. A note on the imaginary part of bispectra. Deep Sea Research Part A. Oceanographic Research Papers 28, 213-222. doi:10.1016/0198-0149(81)90063-7.

Michallet, H., Cienfuegos, R., Barthélemy, E., Grasso, F., 2011. Kinematics of waves propagating and breaking on a barred beach. European Journal of Mechanics - B/Fluids 30, 624-634. doi:10.1016/j.euromechflu.2010.12.004.

Mitsuyasu, H., Kuo, Y.Y., Masuda, A., 1979. On the dispersion relation of random gravity waves. Part 2. An experiment. Journal of Fluid Mechanics 92, 731-749. doi:10.1017/S0022112079000859.

Mouragues, A., Bonneton, P., Lannes, D., Castelle, B., Marieu, V., 2019. Field data-based evaluation of methods for recovering surface wave elevation from pressure measurements. Coastal Engineering 150, 147 - 159. doi:10.1016/j.coastaleng.2019.04.006.

Norheim, C.A., Herbers, T.H.C., Elgar, S., 1998. Nonlinear evolution of surface wave spectra on a beach. Journal of Physical Oceanography 28, 1534-1551. doi:10.1175/15200485(1998)028;1534:NEOSWS ¿2.0.CO;2. 
Ochi, M.K., 1998. Spectral analysis. Cambridge University Press. Cambridge Ocean Technology Series, pp. 13 - 57. doi:10.1017/CBO9780511529559.003.

Padilla, E.M., Alsina, J.M., 2017. Transfer and dissipation of energy during wave group propagation on a gentle beach slope. Journal of Geophysical Research: Oceans 122, 6773-6794. doi:10.1002/2017JC012703.

Peregrine, D.H., Bokhove, O., 1998. Vorticity and surf zone currents, in: Proceedings of the 26th Conference on Coastal Engineering, Copenhagen, Denmark, pp. 745-748.

Phillips, O.M., 1960. On the dynamics of unsteady gravity waves of finite amplitude. Part 1. The elementary interactions. Journal of Fluid Mechanics 9, 193-217. doi:10.1017/S0022112060001043.

Plant, N.G., Holland, K.T., Haller, M.C., 2008. Ocean wavenumber estimation from wave-resolving time series imagery. IEEE Transactions on Geoscience and Remote Sensing 46, 2644-2658. doi:10.1109/TGRS.2008.919821.

Ramamonjiarisoa, A., Coantic, M., 1976. Loi experimentale de dispersion des vagues produites par le vent sur une faible longueur d'action. Comptes rendus hebdomadaires des séances de l'Académie des sciences, Série B 282, 111-113.

Redor, I., Bartélemy, E., Michallet, H., Onorato, M., Mordant, N., 2019. Experimental evidence of a hydrodynamic soliton gas. Physical Review Letters 122, 214502. doi:10.1103/PhysRevLett.122.214502.

Rocha, M.V.L., Michallet, H., Silva, P.A., 2017. Improving the parameterization of wave nonlinearities the importance of wave steepness, spectral bandwidth and beach slope. Coastal Engineering 121, $77-89$. doi:10.1016/j.coastaleng.2016.11.012.

Ruessink, G.B., Michallet, H., Bonneton, P., Mouazé, D., Lara, J.L., Silva, P.A., Wellens, P., 2013. GLOBEX: wave dynamics on a gently sloping laboratory beach, in: Coastal Dynamics '13: Proceedings of the Seventh Conference on Coastal Dynamics, Arcachon, France.

Stive, M.J.F., 1980. Velocity and pressure field of spilling breakers, in: Proceedings of the 17th Conference on Coastal Engineering, Sydney, Australia, pp. 547-566.

Stive, M.J.F., Wind, H.G., 1982. A study of radiation stress and set-up in the nearshore region. Coastal Engineering 6, 1-25. doi:10.1016/0378-3839(82)90012-6.

Stockdon, H.F., Holman, R.A., 2000. Estimation of wave phase speed and nearshore bathymetry from video imagery. Journal of Geophysical Research: Oceans 105, 22015-22033. doi:10.1029/1999JC000124.

Svendsen, I.A., 1984. Mass flux and undertow in a surf zone. Coastal Engineering 8, $347-365$. doi:10.1016/0378-3839(84)90030-9.

Svendsen, I.A., Madsen, P.A., Buhr Hansen, J., 1978. Wave characteristics in the surf zone, in: Proceedings of the 16th Conference on Coastal Engineering, Hamburg, Germany, pp. 520-539. 
Thornton, E.B., Guza, R.T., 1982. Energy saturation and phase speeds measured on a natural beach. Journal of Geophysical Research 87, 9499-9508. doi:10.1029/JC087iC12p09499.

Thornton, E.B., Guza, R.T., 1983. Transformation of wave height distribution. Journal of Geophysical Research: Oceans 88, 5925-5938. doi:10.1029/JC088iC10p05925.

Ting, F.C., Kirby, J.T., 1996. Dynamics of surf-zone turbulence in a spilling breaker. Coastal Engineering 27, 131 - 160. doi:10.1016/0378-3839(95)00037-2.

Tissier, M., Bonneton, P., Almar, R., Castelle, B., Bonneton, N., Nahon, A., 2011. Field measurements and non-linear prediction of wave celerity in the surf zone. European Journal of Mechanics - B/Fluids 30, 635 -641. doi:10.1016/j.euromechflu.2010.11.003.

Tissier, M., Bonneton, P., Michallet, H., Ruessink, B.G., 2015. Infragravity-wave modulation of short-wave celerity in the surf zone. Journal of Geophysical Research: Oceans 120, 6799-6814. doi:10.1002/2015JC010708.

van der Zanden, J., van der A, D.A., Hurther, D., Cáceres, I., O’Donoghue, T., Hulscher, S.J.M.H., Ribberink, J.S., 2017. Bedload and suspended load contributions to breaker bar morphodynamics. Coastal Engineering 129, 74-92. doi:10.1016/j.coastaleng.2017.09.005.

de Wit, F., Tissier, M., Reniers, A., 2020. The relationship between sea-swell bound wave height and wave shape. Journal of Marine Science and Engineering 8, 643. doi:10.3390/jmse8090643. 


\begin{tabular}{cccc}
\hline \hline Test & $H_{s}[\mathrm{~m}]$ & $f_{p}[\mathrm{~Hz}]$ & $\gamma$ \\
\hline A1 & 0.10 & 0.633 & 3.3 \\
A2 & 0.20 & 0.444 & 3.3 \\
A3 & 0.10 & 0.444 & 20 \\
\hline
\end{tabular}

Table 1: Significant wave height $\left(H_{s}\right)$ and peak wave frequency $\left(f_{p}\right)$ for the three tests considered in this study. The peak enhancement $\gamma$ of the JONSWAP spectra characterizes its spectral bandwidth. A value of 3.3 corresponds to broad spectra while a narrow-band spectra is imposed with $\gamma=20$.

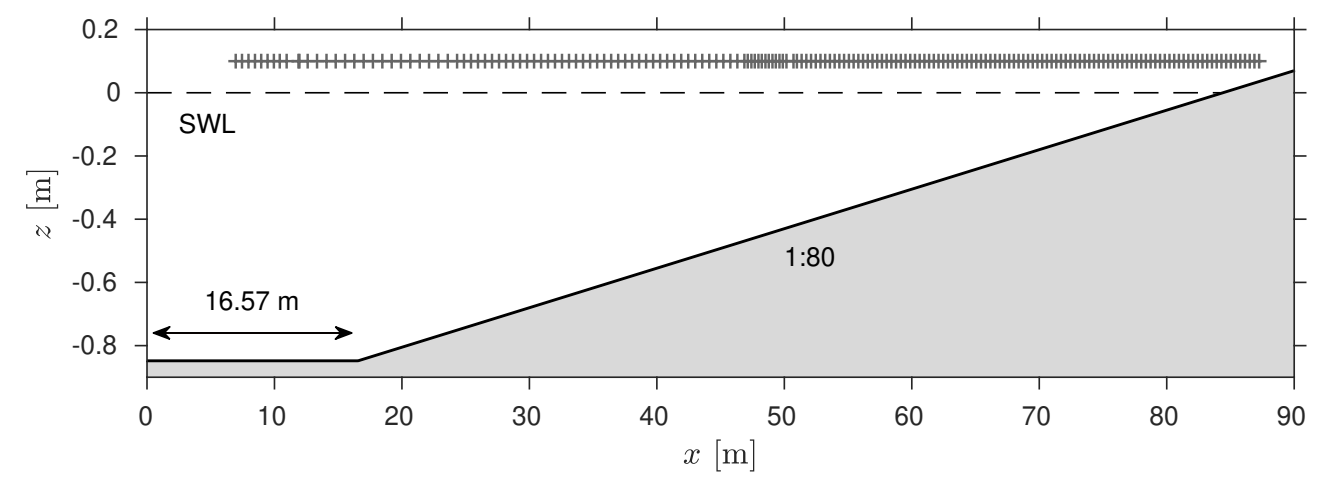

Figure 1: Elevation $z$ of the 1:80 concrete beach against the cross-shore distance $x$ in the Scheldegoot flume during the GLOBEX project. The wave paddle is located at $x=0 \mathrm{~m}$. The grey '+' symbols show the position of the wave gauges across the wave flume.

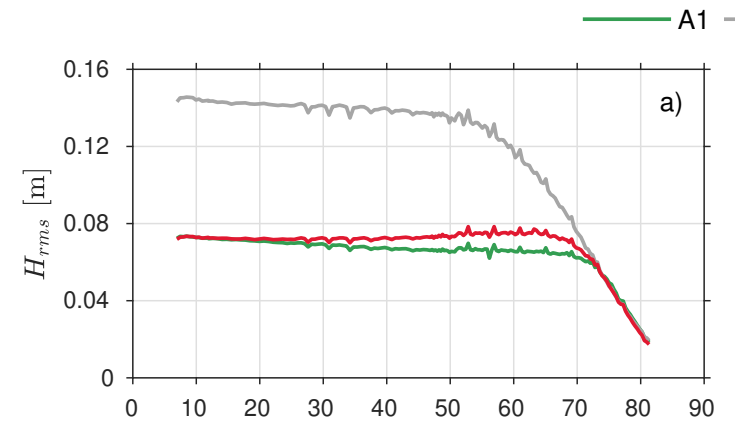

$\mathrm{A} 2 \longrightarrow \mathrm{A} 3$
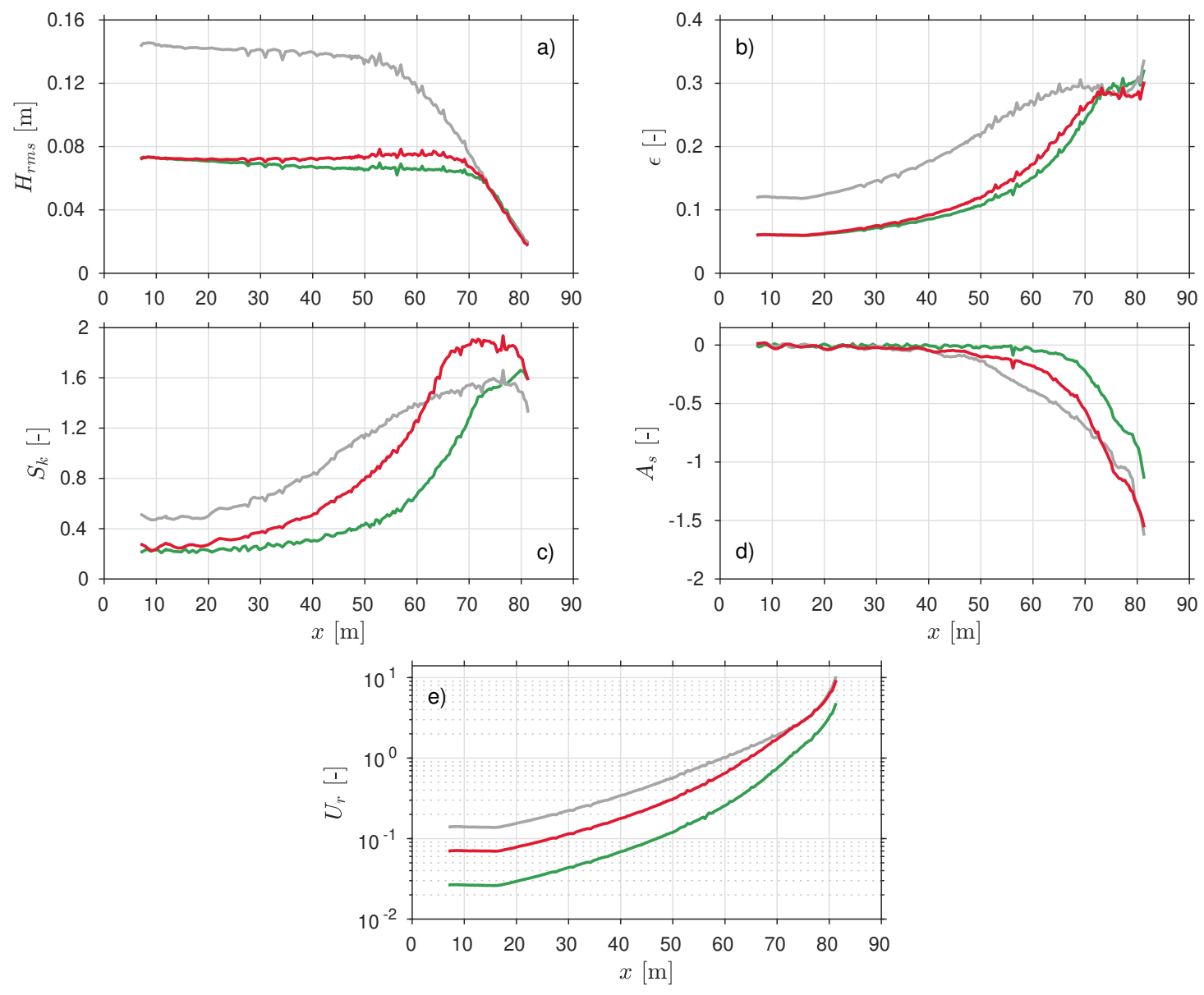

Figure 2: Second and third-order short-wave parameters during tests A1, A2 and A3: a) root-mean square wave height $H_{r m s}$ computed as $\left(8 \overline{\zeta^{2}}\right)^{1 / 2}$; b) corresponding wave amplitude to water depth ratio $\epsilon=H_{r m s} / \sqrt{2} h$; c) wave skewness $S_{k} ;$ d) wave asymmetry $A_{s}$ and e) Ursell number $U_{r}$ computed as $\epsilon / \mu$. Note that these are short-wave parameters, computed using the high-pass filtered surface elevation signal (frequency cutoff at $0.6 f_{p}$ ). 

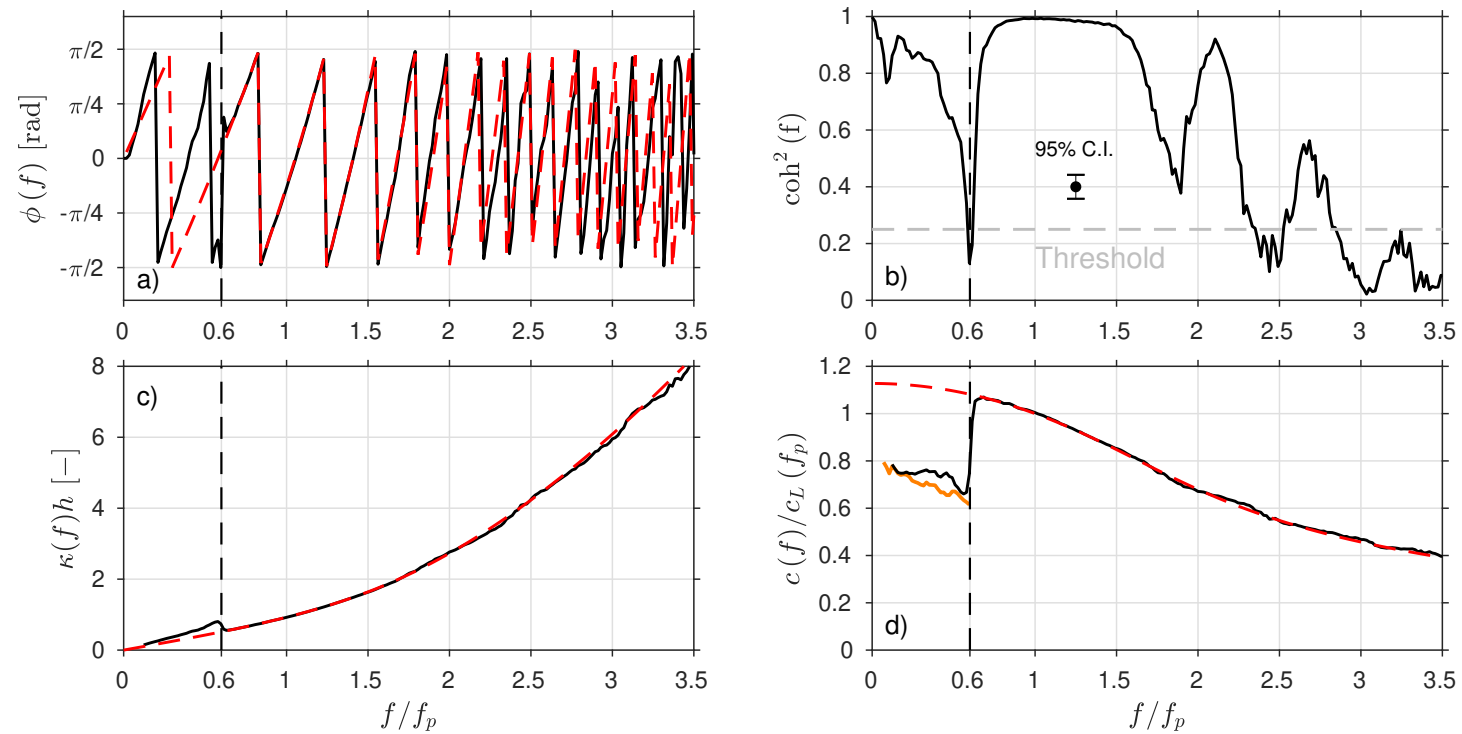

Figure 3: Phase (a), coherence-squared (b), dimensionless wavenumber (c) and phase velocity (d) spectra computed at $x=10.65 \mathrm{~m}$ for case A2. The spacing between the adjacent gauges is $\Delta x=0.93 L_{p}$, with $L_{p}$ the peak wavelength given by the linear wave dispersion relation (Eq. 14). The phase velocity spectrum is normalised by $c_{L}\left(f_{p}\right)$, the phase velocity predicted by linear wave theory at the peak frequency. The red dashed lines in panels a, c and d correspond to the values given by the linear wave dispersion relation. The separation between infragravity and short wave frequencies $\left(0.6 f_{p}\right)$ is shown as dashed black line. In panel b, the gray dashed line corresponds to the coherence-squared threshold used for computing ensemble average spectral estimated of $\kappa$ and $c$. In panel $\mathrm{d}$, the orange line corresponds to the short-wave envelop propagation speed. 
A2
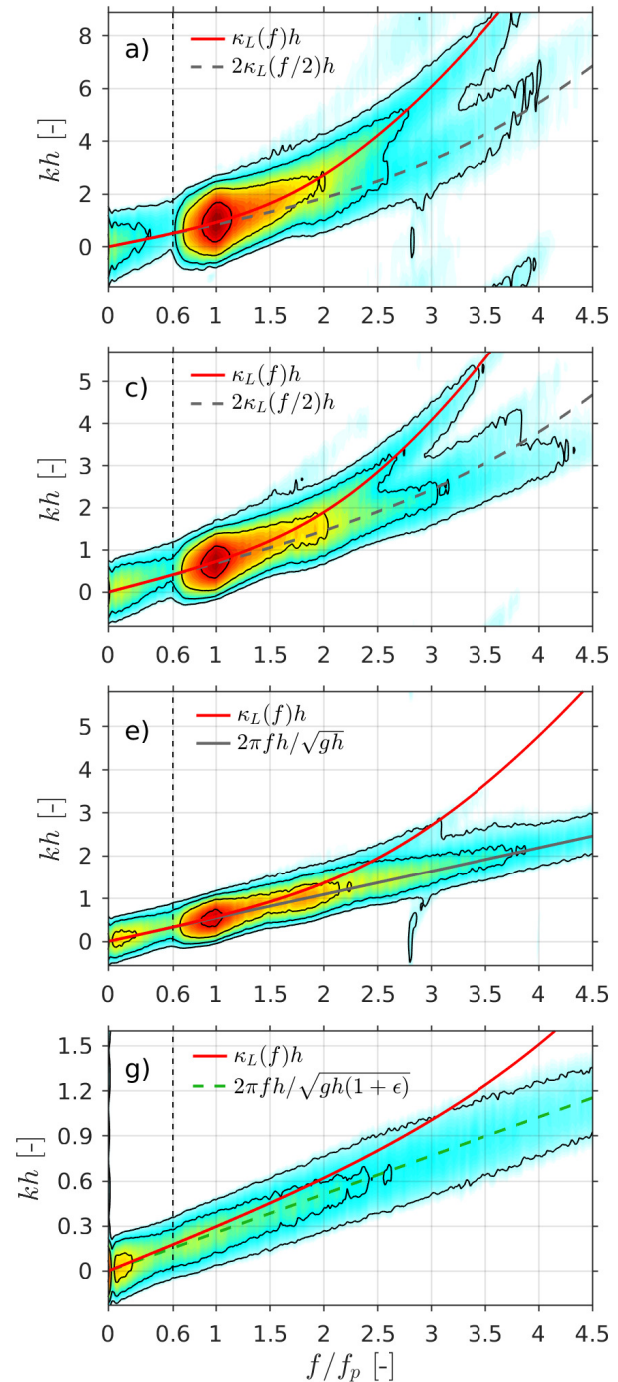

A3
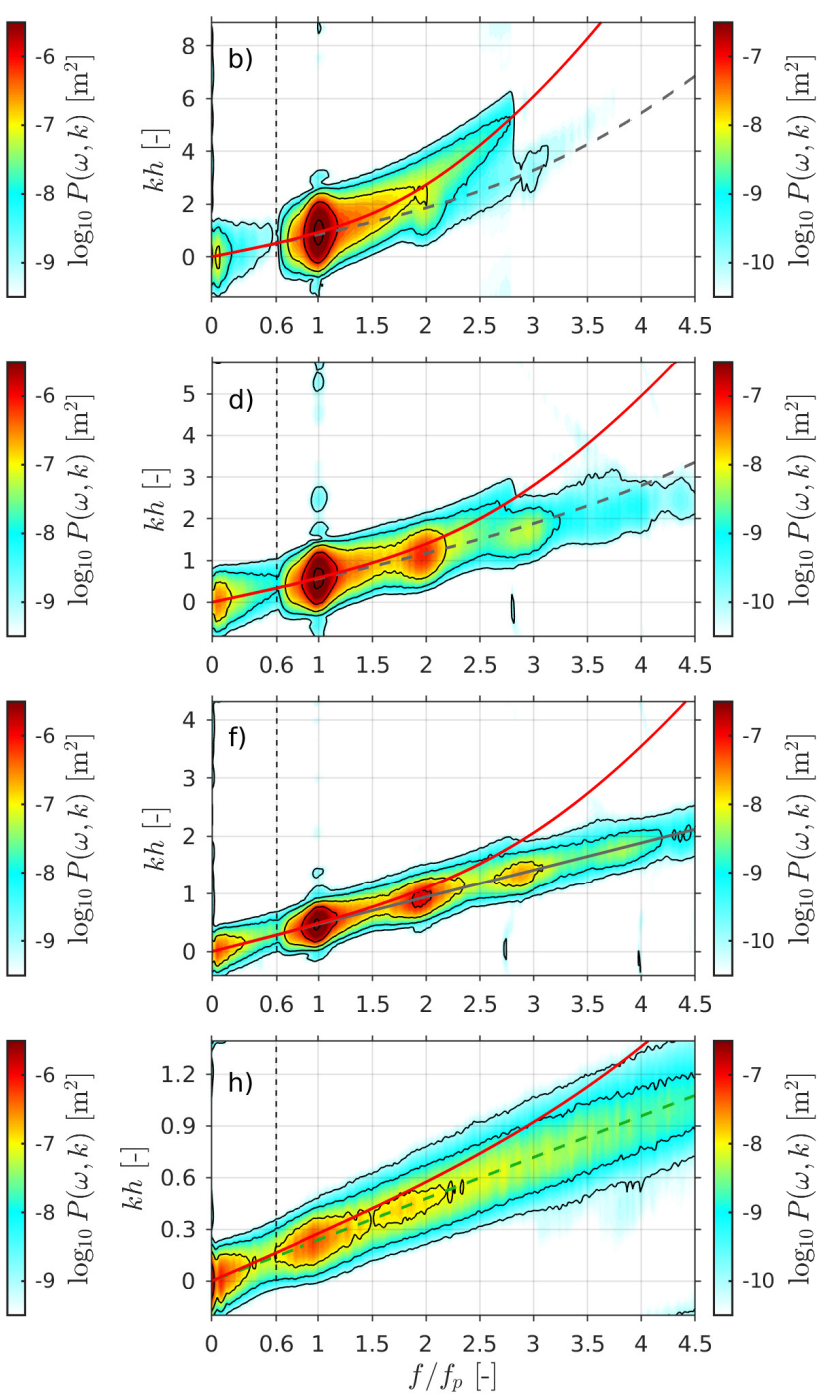

Figure 4: Frequency-wavenumber surface elevation power spectra $P(\omega, k)$ computed for A2 (left) and A3 (right) at the four stages of propagation described in the paper (I: panels a-b; II: panels c-d; III: panels e-f and IV: panels g-h). For A2, these power spectra were computed at I: $x=9.8 \pm 2.8 \mathrm{~m}$; II: $x=38.9 \pm 3.6 \mathrm{~m}$; III: $x=54.5 \pm 3.5 \mathrm{~m}$ and IV: $x=76 \pm 2.4 \mathrm{~m}$. For A3, the cross-shore locations were I: $x=9.8 \pm 2.8 \mathrm{~m}$; II: $x=53.4 \pm 2.4 \mathrm{~m}$; III: $x=62.3 \pm 3.5 \mathrm{~m}$ and IV: $x=77 \pm 2.4 \mathrm{~m}$. Each black contour line correspond to a power of 10 . The separation between infragravity and short-wave frequencies $\left(0.6 f_{p}\right)$ is shown as the dashed black line. In all panels, the red curves correspond to the linear wave dispersion relation. In panels a-d, the dashed grey curves correspond to the dispersion relation for the second harmonic, and was computed based on the assumption that it travels at a similar speed as its principal component. In panels e-f, the grey curve corresponds to the shallow water wave dispersion relation $(c=\sqrt{g h})$ while the dashed green line in panels g-h refer to the modified one $(c=\sqrt{g h(1+\epsilon)})$. 
A2
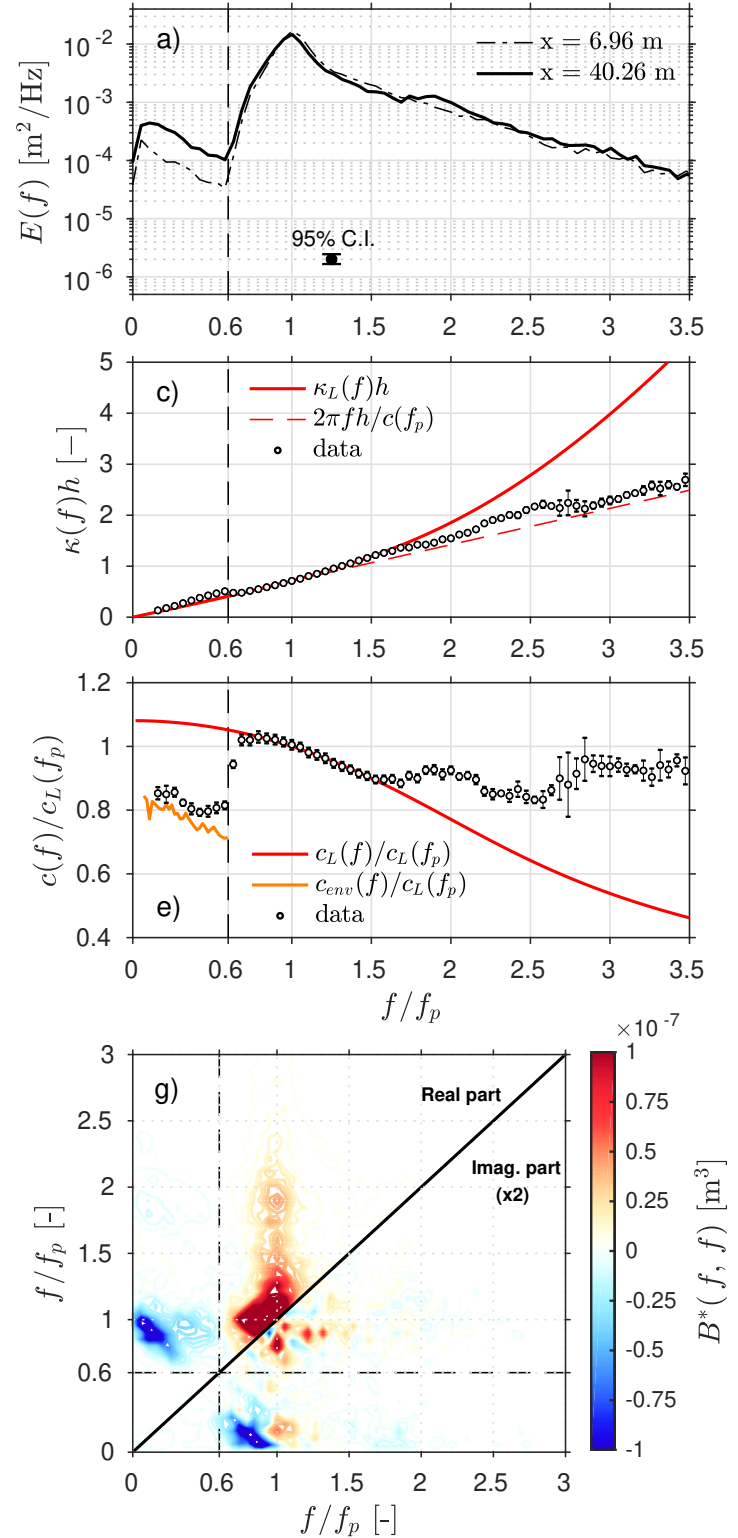

A3
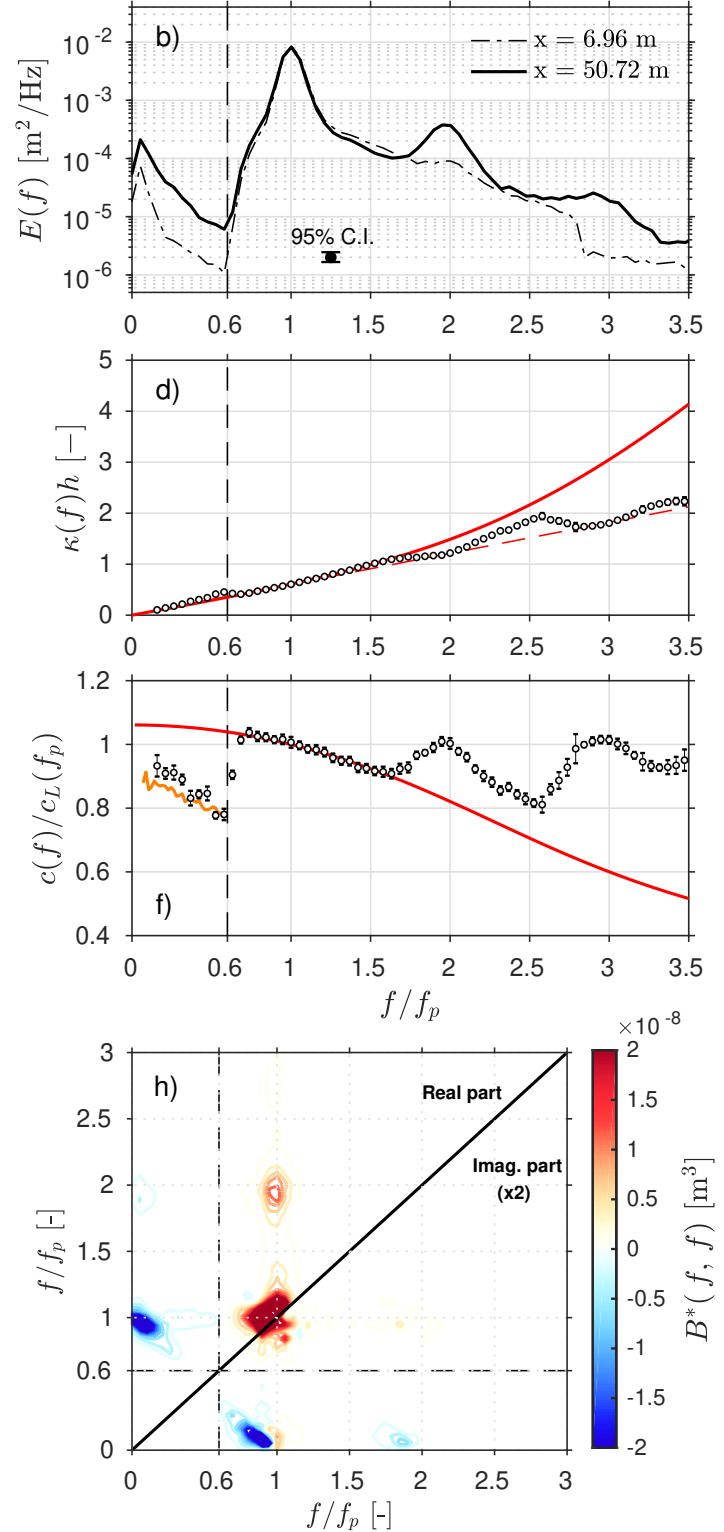

Figure 5: From top to bottom: surface elevation energy density spectra, dimensionless wavenumber spectra, phase velocity spectra normalised by $c_{L}\left(f_{p}\right)$ and bispectra computed at $U_{r} \sim 0.3$ (stage II) for wave tests A2 (left panels) and A3 (right panels). The cross-shore location corresponding to this stage is indicated in panels a and b (cf figure 2e). The wavenumber and phase velocity spectra were computed with five spacing configurations: each point corresponds to the ensemble-averaged value and the error bars correspond to the standard deviation. For readability, only one on three data points are shown. Red lines in panels c-f correspond to values given by the linear wave dispersion relation. The separation between infragravity and short-wave frequencies $\left(0.6 f_{p}\right)$ is shown as the dashed black line. In panels c-d, the red dashed line corresponds to $\kappa(f)=2 \pi f / c\left(f_{p}\right)$. In panels e-f, the orange line corresponds to the short-wave envelop propagation speed denoted $c_{e n v}$. 
A2
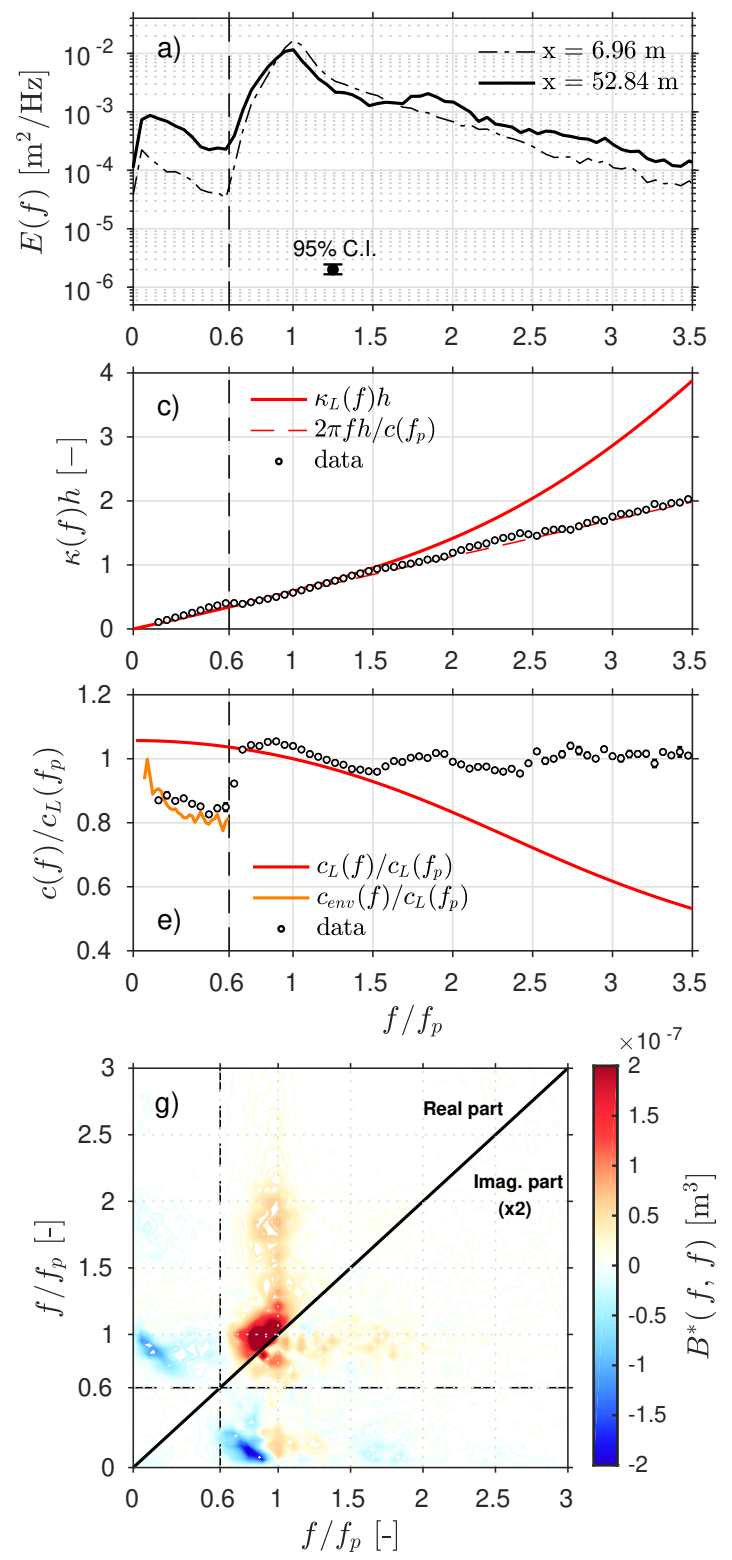

A3
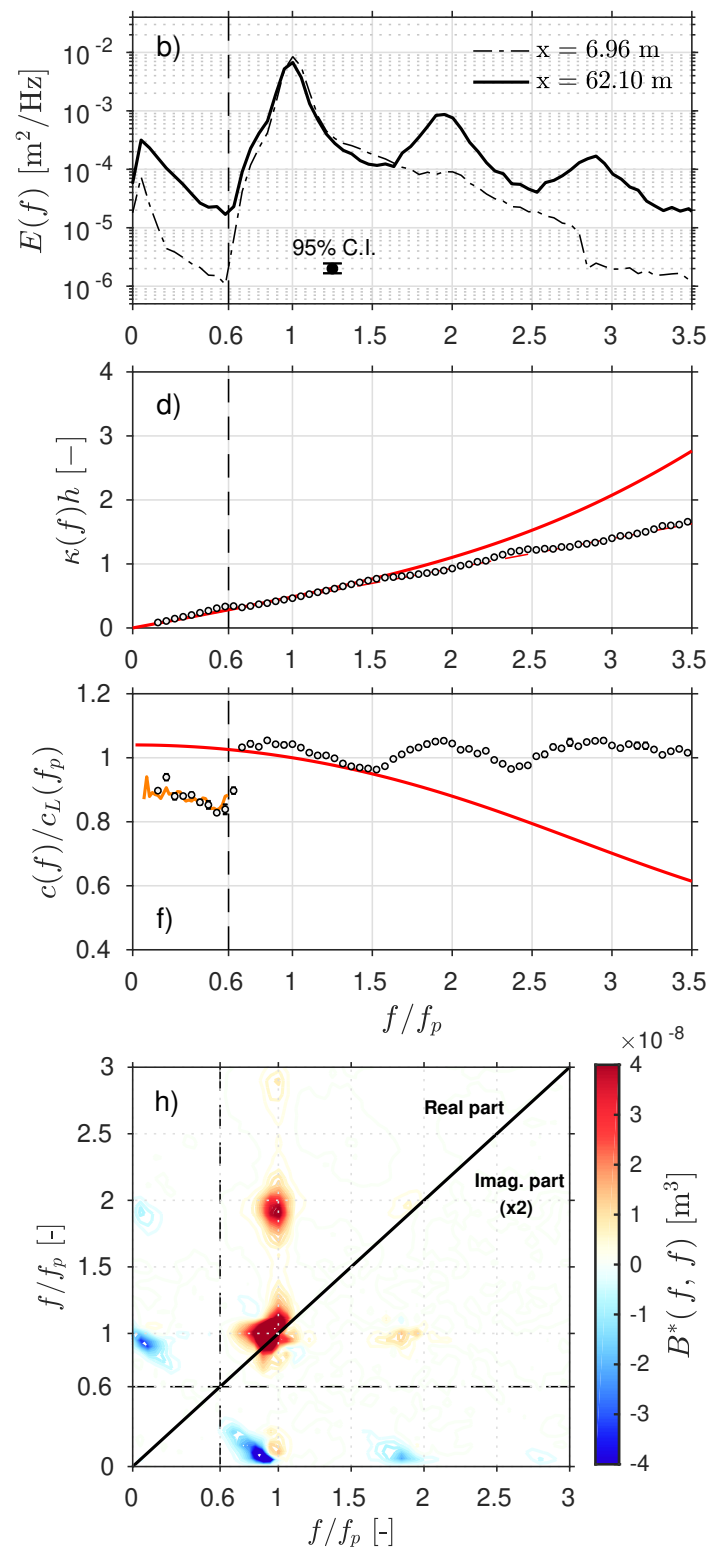

Figure 6: Similar to figure 5, but with $U_{r} \sim 0.7$ (stage III). 
A2
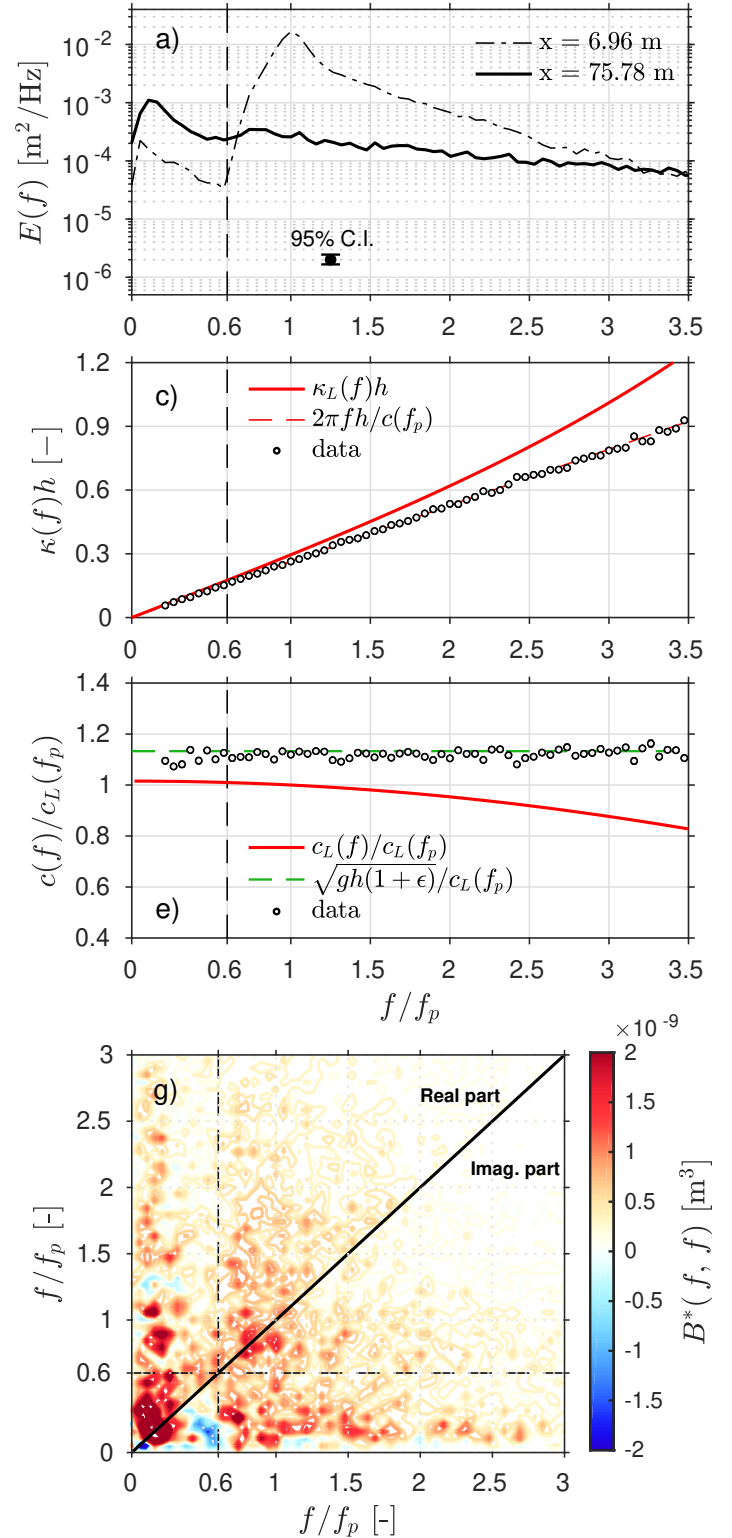

A3
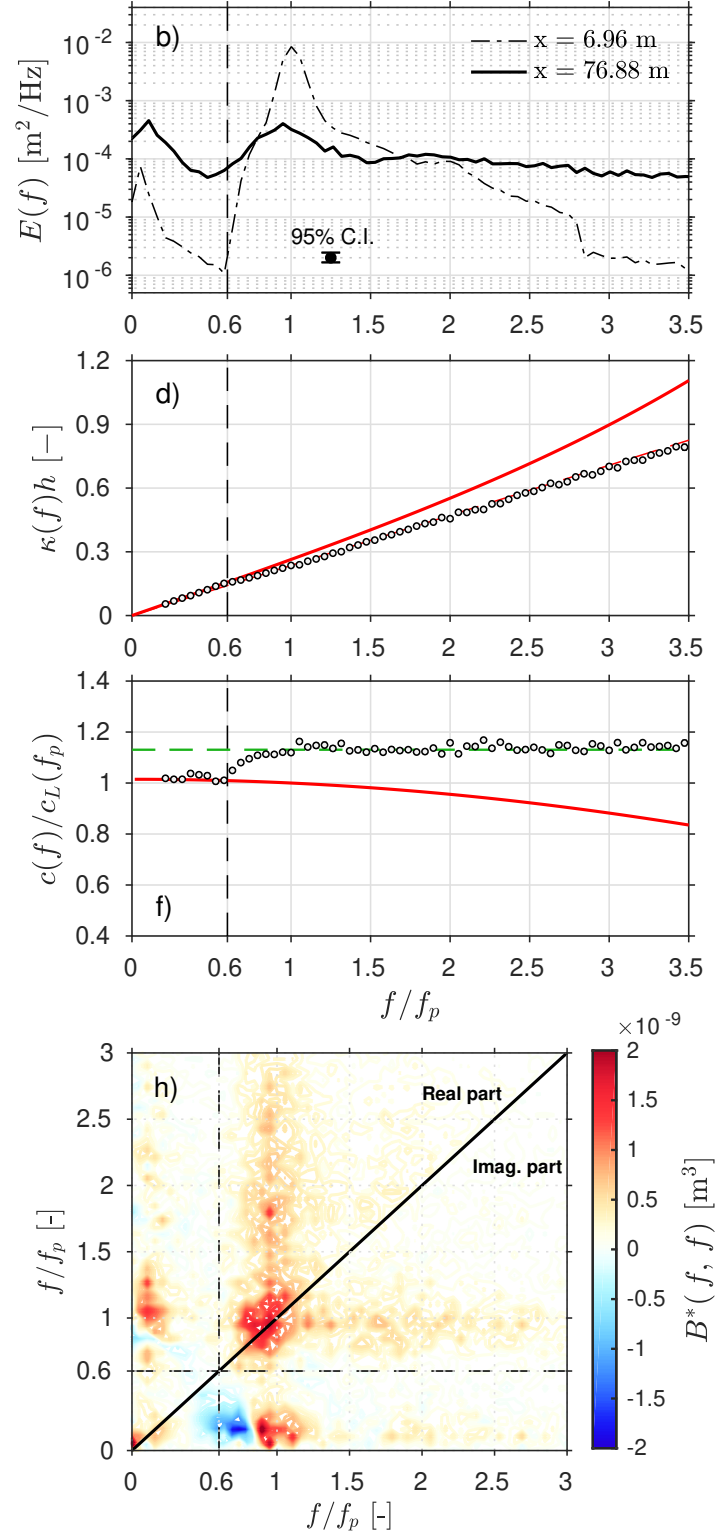

Figure 7: Similar to figure 5, but with $U_{r} \sim 3.2$ (stage IV). Additionally, the green dashed lines in panels e) and f) correspond to the modified shallow water dispersion relation $c=\sqrt{g h(1+\epsilon)}$. 
A2

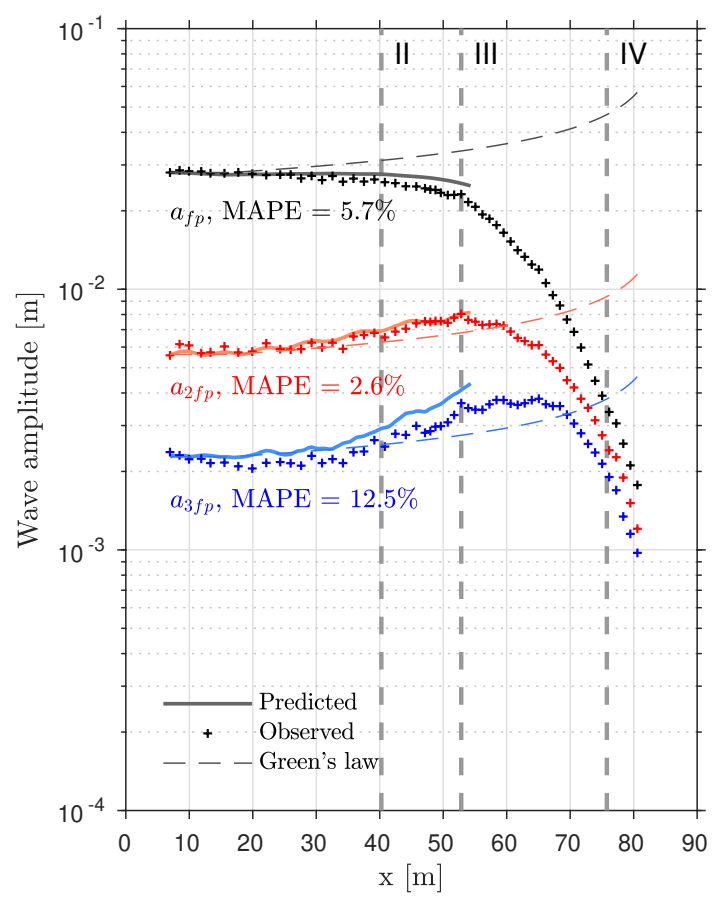

A3

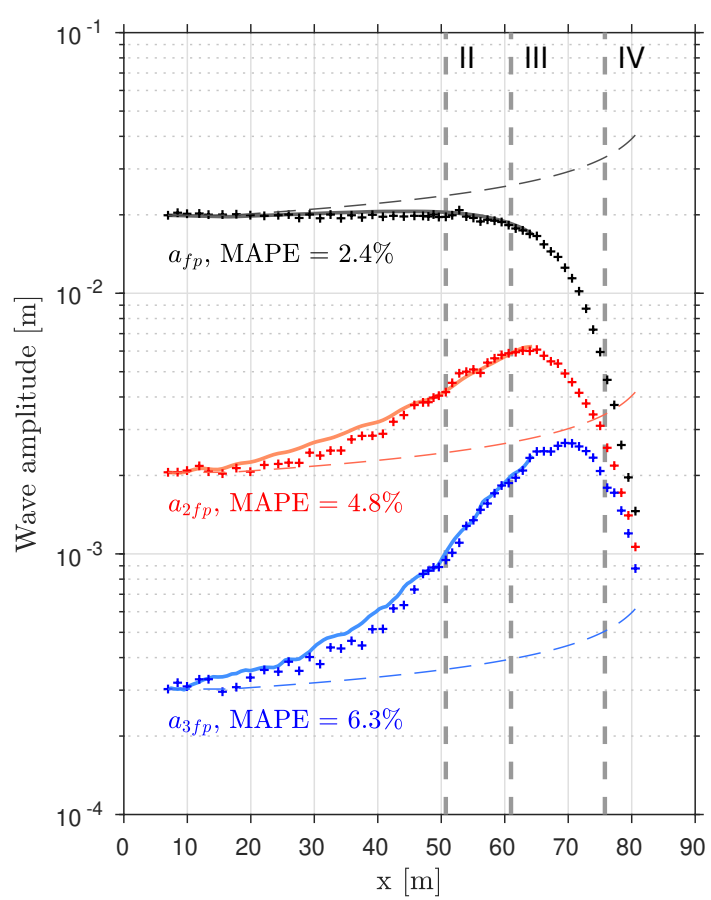

Figure 8: Comparison between observed and predicted (Eq. 13) wave amplitudes at $f_{p}, 2 f_{p}$ and $3 f_{p}\left(a_{f_{p}}, a_{2 f_{p}}\right.$ and $a_{3 f_{p}}$ respectively). The cross-shore locations corresponding to the regimes of propagation discussed in section 4 are indicated by the vertical dashed lines for both wave tests. Since the present modelling approach neglects wave breaking-induced energy dissipation, modelled amplitudes are shown only until the approximate mean breaking point location (slightly after stage III). The amplitude growth predicted with Green's law is also shown.

A2

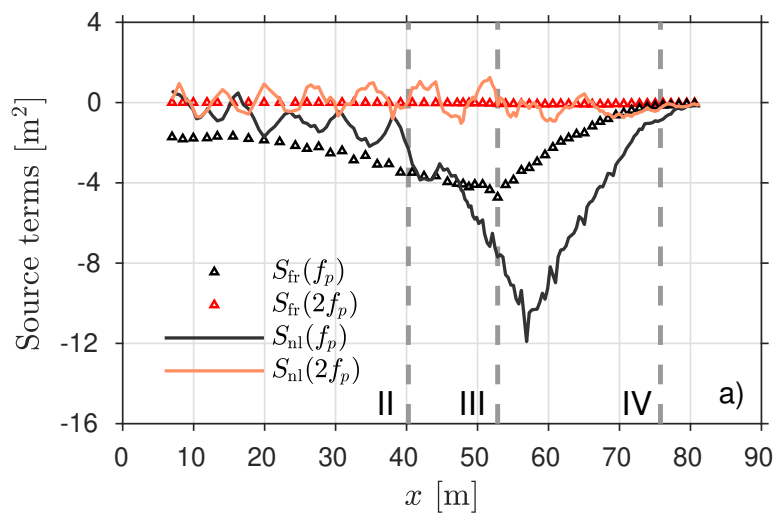

A3

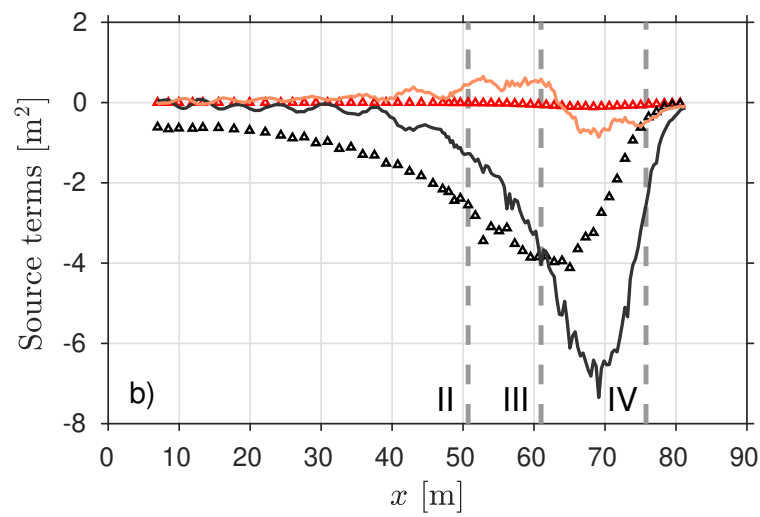

Figure 9: Cross-shore variation of the source terms for the non-linear energy exchanges between triads and for the bottom friction, used in Eq. 13. Only values at the peak frequency $\left(f_{p}\right)$ and the second harmonic $\left(2 f_{p}\right)$ are shown. The cross-shore locations corresponding to the regimes of propagation discussed in section 4 are indicated by the vertical dashed lines for both wave tests. 


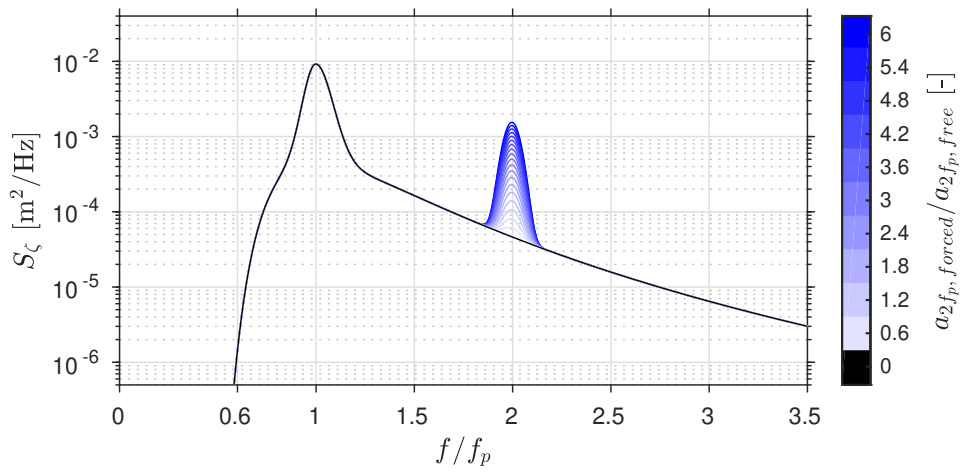

Figure A1: Energy density spectra imposed at a fictive $x_{1}$ position, with different ratio of forced to free second harmonic amplitude. In the absence of forced components, the forcing corresponds to a JONSWAP spectrum with parameter similar to A3 (see Table 1).
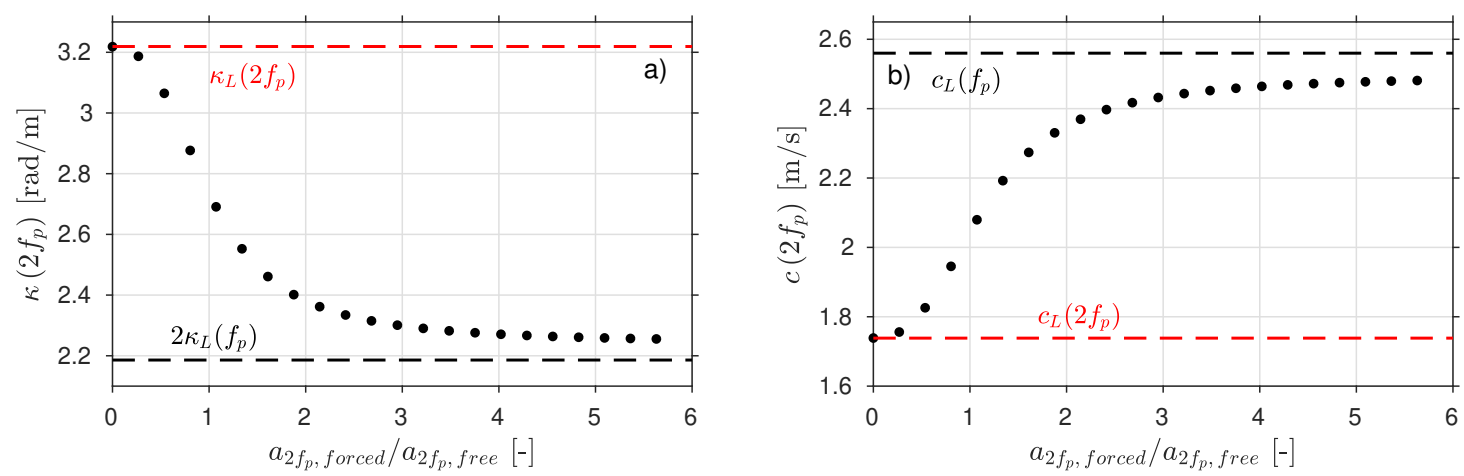

Figure A2: Wavenumber (a) and phase velocity (b) spectra computed on the synthetic surface elevation timeseries for varying ratio of forced to free second harmonic amplitudes.

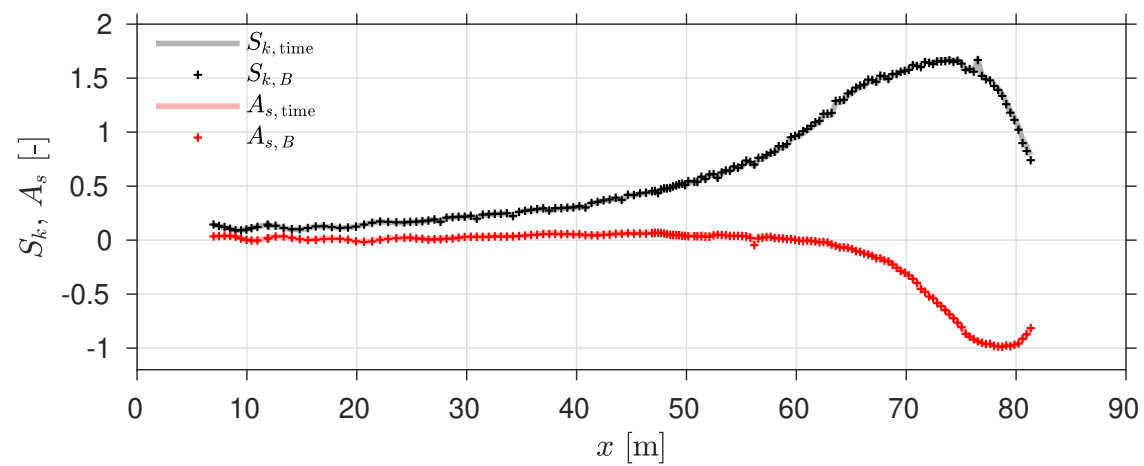

Figure B1: Cross-comparison of third-order wave parameters during A3 computed using the statistical (Eq. 1-2, 'time' subscript) and the bispectrum (Eq. B.1-B.2, 'B' subscript) definitions. No separation between infragravity and short wave frequency bands or between incoming and outgoing wave fields was performed for this comparison. 
A2

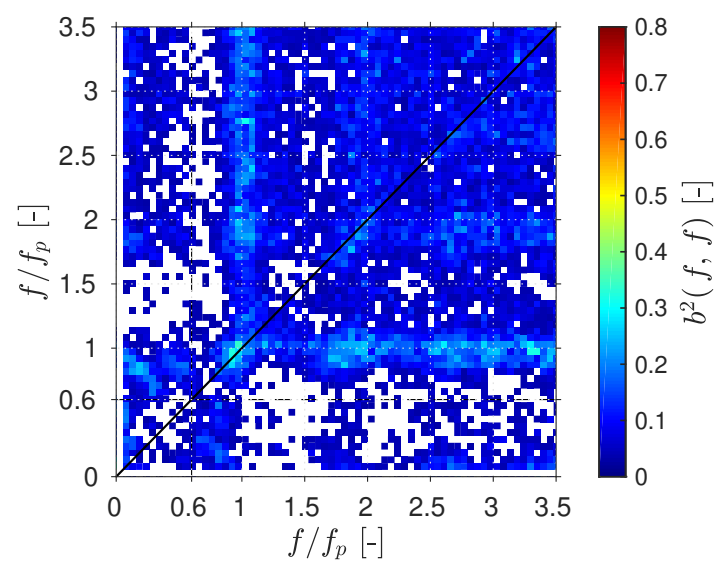

A3

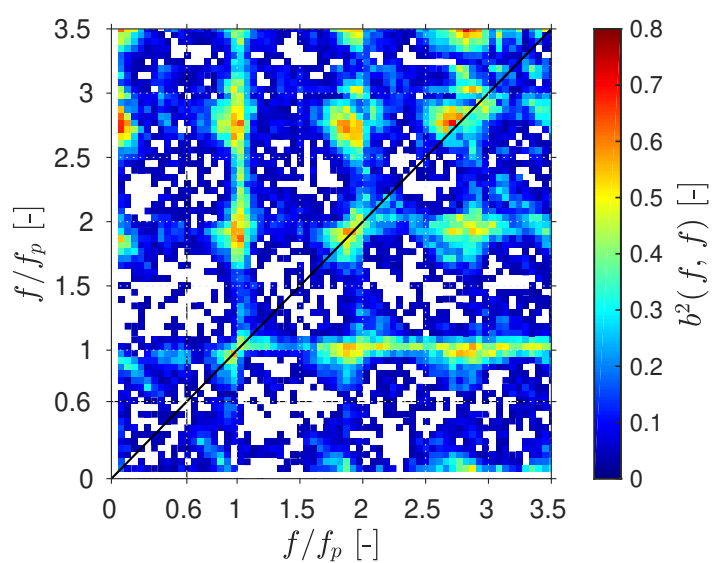

Figure C1: Squared bicoherence $b^{2}$ computed using the definition of Hagihira et al. (2001) for wave test A2 (left) and A3 (right). The octant above the 1:1 diagonal corresponds to the bicoherence for stage II while stage III is that under the diagonal. Due to the symmetric properties of the bispectrum, only one octant is shown for each stage. The separation between infragravity and short-wave frequencies $\left(0.6 f_{p}\right)$ is shown as the dashed black line. Only bicoherence values greater than the $95 \%$ significance level as defined by Haubrich (1965) ( $b_{95 \%}^{2} \geq 6 /$ d.o.f., with d.o.f. the equivalent number of degrees of freedom) are shown. 